\title{
Influence of bulb sizes at planting on growth and development of the common hyacinth and the lily
}

\author{
Addai, I. $\mathrm{K}^{1}$ and Scott, $\mathrm{P}^{2}$ \\ ${ }^{1}$ Corresponding author's address: Flat 38F, Park Village, Sussex University, Falmer, \\ Brighton, BN1 9RD (UK) or The Agronomy Department, UDS, P. O. Box 1350, Tamale, \\ Ghana. E-mail: isaackwaheneaddai@yahoo.com; Tel: 00447919610749. \\ ${ }^{2}$ Biology and Environmental Science Department, University of Sussex, Falmer, Brighton, UK.
}

\begin{abstract}
Studies were carried out at the Plant Stress Unit of the University of Sussex, UK to determine the effects of bulb size at planting the on the growth and development of flower bulbs using hyacinth and the lily as test plants. Results of the studies indicated that bulb size at planting influenced vegetative growth and all other parameters measured both in hyacinth and the lily. As the size of the planted bulb increased, parameters measured also increased in proportion to the size of the planted bulb. In hyacinth, however, the possession of a large bulb had little advantage, overall, to growth and development because after the $50 \mathrm{~g}$ bulb size, the results look markedly similar. Probably, the extra carbohydrate reserves possessed by the large bulbs ( $>50 \mathrm{~g}$ bulb sizes) might be useful to the bulb later during their life cycle for survival during unfavourable environmental conditions. In general plants produced from large bulb grew faster to complete their life cycle than those produced from medium and small bulbs but senescence occurred earlier in the former than the latter.
\end{abstract}

Keywords: Common hyacinth, the lily, growth and development, bulb size at planting, flower bulb.

\section{INTRODUCTION}

The common hyacinth, Hyacinthus orientalis, is a spring-flowering bulb and belongs to the family Hyacinthaceae and genus Hyacinthus. It is a horticulturally important plant, and native of the West and Central Asia. Hyacinth is used in the perfumery industries because an essential oil is extracted from its flowers for the manufacture of perfumes (Usher, 1974). The diploid forms of hyacinth plant have 16 chromosomes of 5 different types and there are also triploids $(3 n=24)$ and a large number of heteroploid (Rees, 1972). Several species are cultivated, but the most well known is Hyacinthus orientalis L. which was first developed by artificial selection in Turkey and subsequently in Holland (Gorer, 1970). The Lily is a summer flowering bulb and also belongs to the family Liliaceae, order Liliales and the genus is Lilium. Bulbs of Lilium species are starchy, and some are edible as root vegetables. Some Lilium species are commercially grown in China as food. They are eaten especially in the summer, because they have the ability to reduce internal heat. They may be reconstituted and stir-fried, grated and used to thicken soup, or processed to extract starch (Chittendon, 1956; Huxley, 1992). Bulbs of Tiger Lily, Lilium lancifolium Thunb, may be cooked and eaten
(Hedrick, 1972; Fox, 1985; Grieve, 1984). In general, flower bulbs are cultivated as ornamental geophytes and this is the chief economic value of these plants.

In geophytic and other vegetatively propagated plants, growth and development is influenced by the amount of stored reserves present in the tuber, corm, rhizome, or bulb at the time of planting (Bremner and El Saeed, 1963; Burton, 1966; Rees, 1969). Afonja (1967) found higher yields of tubers from higher sets weights of white yam (Dioscorea rotunda) and Enyi (1967) obtained higher yields of cocoyam from plants grown from larger sets. According to Watad et al. (1999), flowering in Aconitium, a member of family Ranunculaceae, was highly dependent on tuber weight. In this species, tuber size of 30-40 g produced flowers of good quality whereas plants produced from tubers of $5 \mathrm{~g}$ or less did not flower. Also, plants from larger tubers produced more tubers, whereas smaller ones recorded a higher growth rate. Hidekazu et al. (1998) compared different seed tuber sizes of yam on growth and yield of the crop as well as sprouting and biomass reduction associated with the seed tuber sizes. They reported that the size of the yam seed tuber used for propagation influenced both the degradation of reserves stored in the tuber and the eventual growth and development of that 
plant because the characteristics of the aboveground organs of yam: stem length, number of branches, total leaf area and the length as well as the weight of the newly developed tubers positively correlated with the initial seed tuber size. In Brodiaea, however, Han et al. (1991) stated that the percentage of the corm that flowered, and flower quality was independent of the mother corm size used for propagation, but rather it was the size of the apical meristem that determined the quality of the flowers produced.

Flower bulbs are generally cultivated for their flower production, and there is a relationship between the reserves of the bulb scales and flower production (Rees, 1985). Therefore, large bulbs are expected to have higher vegetative growth and development than small bulbs because the former have a relatively higher reserves and volume of scales than the latter. However, information on the particular bulb sizes that will produce good quality flowers and bulb yield in hyacinth or the lily has not been documented in the literature, but such data is necessary because it would allow growers to select the right sizes of these species for propagation in order to achieve products which are of good quality for consumers. Studies were thus conducted at the University of Sussex with a view to studying the influence of various bulb sizes at planting on growth and productivity of flower bulbs using the common hyacinth and the lily as test plants.

\section{MATERIALS AND METHODS}

Planting: Hyacinth bulbs whose average fresh weights were 10, 20, 30, 40, 50, 60, 70 and $80 \mathrm{~g}$ were used whilst the lily bulbs were grouped into the following sizes: 10-19, 20-29, 30-39 and 40-49 g. In plant species, 4 bulbs per pot were planted in plastic pots, the capacity of each was $0.01 \mathrm{~m}^{3}$. Prior to planting, pots were filled with compost and perlite mixture in a ratio of $2: 1$ by volume. The units were arranged randomly on greenhouse benches using randomised complete block design.

Methods of data collection: Leaf length was measured as length of the leaf from the base to the leaf tip, whilst leaf width was measured as the distance at the middle section of the leaf where it is broadest. Total leaf length and width were recorded as the sum of all the individual leaf lengths and widths for one particular plant. Total leaf area was deduced from leaf length and width as described below under section 2.2.1.

Determination of leaf area: The method employed in the determination of leaf area was modified from the procedure used by Darkwa (2008). The leaves of 40 plants from both species grown in the greenhouse were obtained through a destructive sampling. The product of the length and broadest part of the leaf (width) was recorded as measured leaf area (MLA) for all these leaves. Then, outlines or shapes of these leaves were sketched on A-4 papers, and cut out with a pair of scissors. These pieces of papers were weighed separately using an electronic weighing balance and the weight of each piece of paper was recorded as leaf paper weight (LPW). The lengths and widths of three of such A-4 papers were multiplied in order to get areas of these A-4 papers, and the average area of the three A-4 papers was divided by the average weight to get a constant value. This constant value was multiplied by the weight of each piece of paper (LPW) obtained by cutting out the leaf outlines and the value recorded as the leaf true area (LTA). A graph of LTA was plotted against MLA and a line of best fit was made to pass through the points, and the gradient of this line was taken as the leaf area constant value for each species as shown in Figure 1. Thus for instance, the leaf area constant value for hyacinth was 0.9543 whilst that of lily was 0.8921 . Therefore in the case of hyacinth, the leaf area (LA) for one particular leaf was calculated as MLA x 0.9543, and the total leaf area for the whole plant is the sum of (MLA x 0.9543) of all leaves on one particular plant. In the lily, four leaves from different positions of each plant that is lower part of the shoot, middle section, towards the top of the plant and at the shoot tip were considered in the computation of (MLA $x$ 0.8921), and the average value was obtained. Thus the total leaf area for the lily was calculated as the average of (MLA x 0.8921) $x$ total number of leaves on the plant at that particular time.

\section{Inflorescence development:}

Inflorescence development was measured in terms of inflorescence length, inflorescence diameter, inflorescence stalk diameter, peduncle length and number of florets. In hyacinth, inflorescence height was recorded as the distance from the soil surface level of each plant to the tip of the inflorescence. In the lily, it was measured as the distance from soil surface to the tip of the tallest flower. Inflorescence length was taken as the inflorescence height minus the length of the stalk holding the inflorescence. Peduncle length or the length of the flower stalk was measured in the lily as the length from the point of attachment of the flower from the shoot to the base of the flower. Inflorescence stalk diameter was 
measured in hyacinth as the growth in girth of the stalk holding the inflorescence that is, it is a measure of thickness of the inflorescence stalk. Similarly, inflorescence diameter was recorded as a measure of inflorescence thickness before the opening of the florets, and this was measured as the growth in girth of the inflorescence in hyacinth. All these parameters were recorded using a ruler but the number of florets was recorded by counting them after they were fully opened.

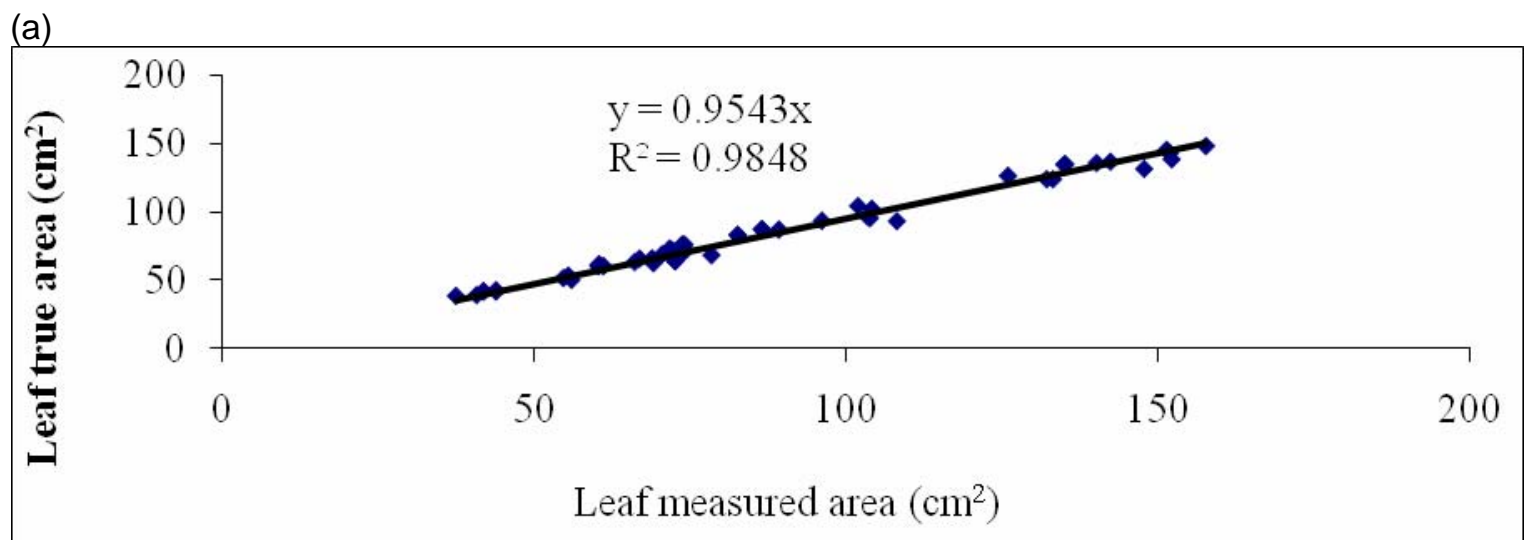

(b)

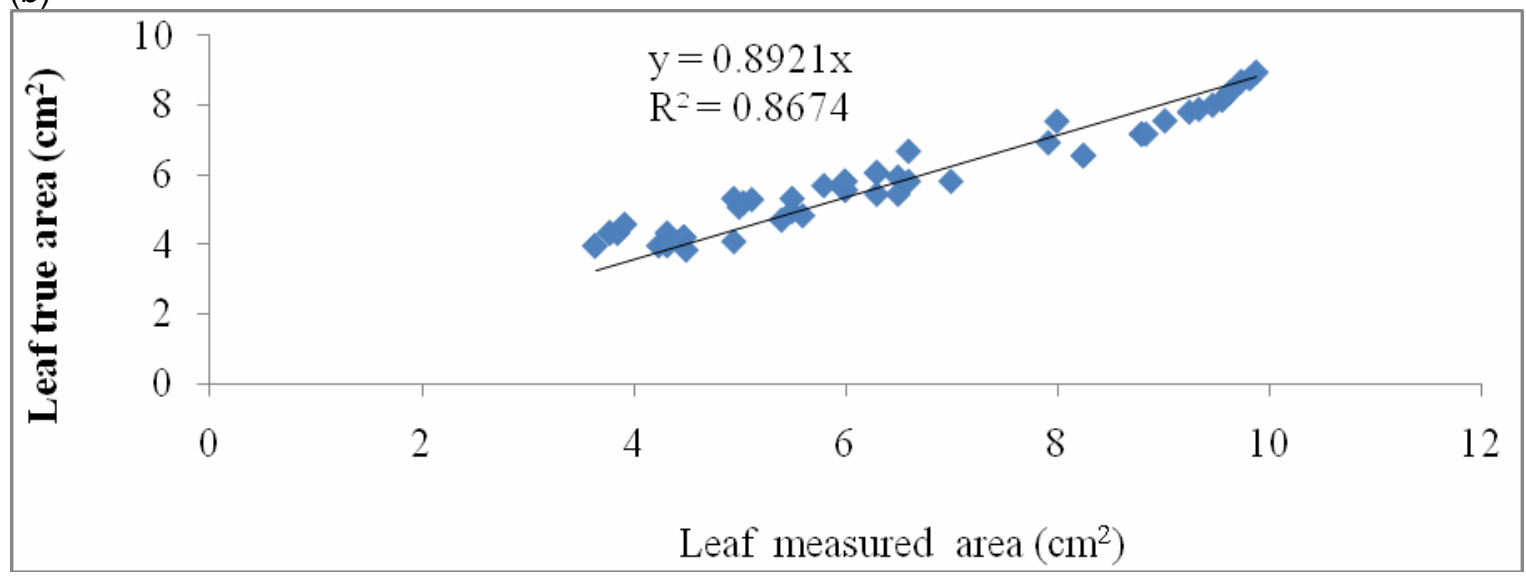

Fig 1: Determination of leaf area constant for (a) the common hyacinth, and (b) the lily.

Bulb fresh weight, gain in fresh weight and plant vigour rating: The fresh weights of bulbs were recorded with an electronic weighing balance as the weight of the bulbs prior to planting. At harvesting, gain in fresh weight was simply measured as the harvest weight less the initial fresh weight at planting. Vigour rating of plants was made by scoring the overall vigour or health of the plant stand. Considerations were given to the general plant performance and attributes such as leaf greenness, growth rate, wilting or drooping of leaves, flower development and canopy architecture. Plant weakness and plant height were also of paramount importance in scoring. In this measurement, a scale of $1=$ very weak, $2=$ weak, $3=$ moderately healthy, 4 $=$ healthy and $5=$ very healthy was used. Data on scoring were log transformed prior to ANOVA, but were back transformed for easy comparison among treatment means.

Chlorophyll content, photosynthetic rate and stomatal conductance: Leaf chlorophyll concentration was measured with a SPAD chlorophyll meter (Minolta SPAD-502) that gives a relative index of leaf concentration. The instrument was first calibrated and clipped to three points that is the lower part, the middle portion and towards the tip of the leaf whose chlorophyll content was desired. In hyacinth, 
the first three leaves from outside were considered whilst in the lily, six leaves from different positions of the plant were involved that is two from the lower part of the shoot, two from the middle section and two from the topmost part of the shoot system of each plant. Averages were computed for each plant prior to the analysis of this data. Using the infrared gas analyser (Ciras-1 PP Systems), photosynthetic rate and stomatal conductance were determined between the hours of $12: 00$ and $15: 00$ at the prevailing solar radiation. A known area from the leaves used for chlorophyll measurement was clipped with the cuvette of the IRGA, and measurements were made once the leaves had acclimatised to the conditions.

\section{RESULTS}

Hyacinth: In general, the parameters measured increased with increasing bulb size at planting. Vast differences existed in leaf growth of plants obtained from the smallest and largest bulb sizes. For instance, in the case of plants obtained from the $10 \mathrm{~g}$ bulb size, leaf width, length and area increased from $3.95 \mathrm{~cm}, 7.90 \mathrm{~cm}$ and $6.01 \mathrm{~cm}^{2}$ to $10.99 \mathrm{~cm}, 132.58$ $\mathrm{cm}$ and $214.37 \mathrm{~cm}^{2}$, respectively, from 12 to 24 weeks after planting (Fig $2 \mathrm{a}$ and $\mathrm{b}$; Fig $3 \mathrm{a}$ ). These increases represented relative growth rates of 0.084 $\mathrm{cm}$ day $^{-1}, 1.484 \mathrm{~cm}$ day $^{-1}$ and $2.480 \mathrm{~cm}^{2}$ day $^{-1}$, respectively. In the case of plants obtained from the largest bulb (80 $\mathrm{g}$ size), leaf width, length and area increased from $9.79 \mathrm{~cm}, 13.83 \mathrm{~cm}$ and $20.93 \mathrm{~cm}^{2}$ at 12 weeks after planting to $28.13 \mathrm{~cm}, 215 \mathrm{~cm}$ and $689.89 \mathrm{~cm}^{2}$, respectively, at 24 weeks after planting, giving relative growth rates of $0.219 \mathrm{~cm}^{-1 a y^{-1}}, 2.394$ $\mathrm{cm}$ day $^{-1}$ and $7.964 \mathrm{~cm}^{2}$ day $^{-1}$, respectively. Also, the chlorophyll content of plants obtained from the large bulbs was generally higher than those of the small bulbs (Fig $3 b$ ) but the reduction in this parameter particularly from 16 to 22 weeks after planting was higher in the case of the large bulbs than plants from the small bulbs. For example, in the case of plants produced from the small bulbs $(10,20$ and $30 \mathrm{~g}$ sizes) the leaf chlorophyll concentration generally increased from 12 to 22 weeks after planting whilst for the large bulbs (60-80 g sizes), chlorophyll content increased from 12 to 18 weeks after planting, and dropped from this point to 22 weeks after planting. The chlorophyll content of plants from the medium bulb (40-50 g) also dropped at 18 weeks after planting. Thus for example, whilst there was no reduction in chlorophyll content of plants from the small bulbs (10-30 $\mathrm{g}$ sizes), in the case of those from the $40 \mathrm{~g}$ bulb size, chlorophyll content dropped from 69.48 to 62.31 spad units from 16 to 22 weeks after planting, representing $10.31 \%$ reduction in chlorophyll whilst in the case of those from the 70 and the $80 \mathrm{~g}$ bulbs, leaf chlorophyll concentration reduced from 75.14 to 51.97 spad units and from 75.75 to 58.02 spad units, respectively, and these represents $30.8 \%$ and $23.41 \%$ reduction in chlorophyll. Inflorescence height, inflorescence length, inflorescence diameter and inflorescence stalk diameter (flower quality) all followed the distribution pattern exhibited by leaf growth, that is all these parameters increased with increasing bulb size at planting (Fig 4-5). The rate of growth in height of the inflorescence was gradual from 14 to 18 weeks after planting but very sharp between 20 and 22 weeks after planting. Also, between 20 and 22 weeks after planting, the plants obtained from 60,70 and 80 $\mathrm{g}$ bulbs recorded similar values of inflorescence height and length, (Fig 4a and b). Similarly, there was no significant difference in inflorescence diameter for plants produced from 50, 60, 70 and $80 \mathrm{~g}$ bulbs between 20 and 22 weeks after planting. There was a vast difference in terms of inflorescence development for the plants obtained from the 10 and $80 \mathrm{~g}$ bulbs. For example, from 14 to 22 weeks after planting, plants obtained from the $10 \mathrm{~g}$ bulb size recorded values of inflorescence height and inflorescence length of $1.67-17.67 \mathrm{~cm}$ and $1.17-3.43 \mathrm{~cm}$, respectively, whilst those obtained from the $80 \mathrm{~g}$ bulb size had inflorescence height and inflorescence length values of $4.67-37.67 \mathrm{~cm}$ and $6.67-16.33 \mathrm{~cm}$, respectively. Similarly, very few florets were observed from plants produced from the small bulbs as compared to those of the large ones. Average number of florets ranged from 2.33 in the case of those from the $10 \mathrm{~g}$ bulb size to 12.33 for those belonging to the $40 \mathrm{~g}$ bulb size, whilst in the case of plants produced from the large bulbs $(60-80 \mathrm{~g})$, number of florets ranged from 55 to 73 (Fig 6a). The fresh weight of bulblets generally increased with increasing bulb size at planting (Fig 6b). Similarly, the fresh weight of the mother bulb at harvest followed the same distribution pattern as number of florets (Fig 7) but the percentage gain in weight at harvest was inversely related to bulb size at planting. In general, the measurements made of hyacinth in this experiment revealed that, after the 50 or $60 \mathrm{~g}$ bulb size, values of parameters appeared to be similar in magnitude, and in most cases, differences were not significant above the $50 \mathrm{~g}$ bulb size. This means that, the $60 \mathrm{~g}, 70 \mathrm{~g}$ and the $80 \mathrm{~g}$ bulb sizes recorded similar values of either vegetative growth or flower quality, and the possession of large bulb size, in 
hyacinth, seemed to be of little advantage to the plant

(a)

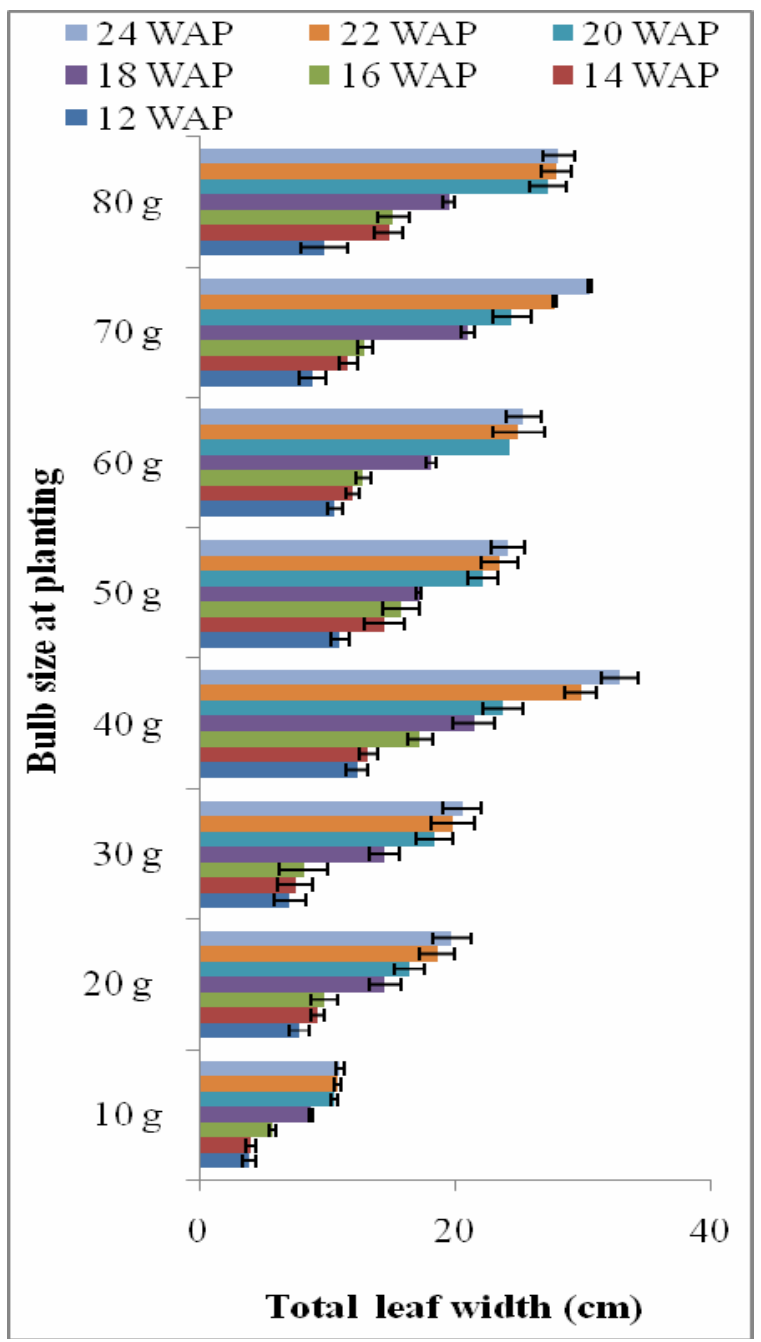

in terms of growth and flower production.

(b)

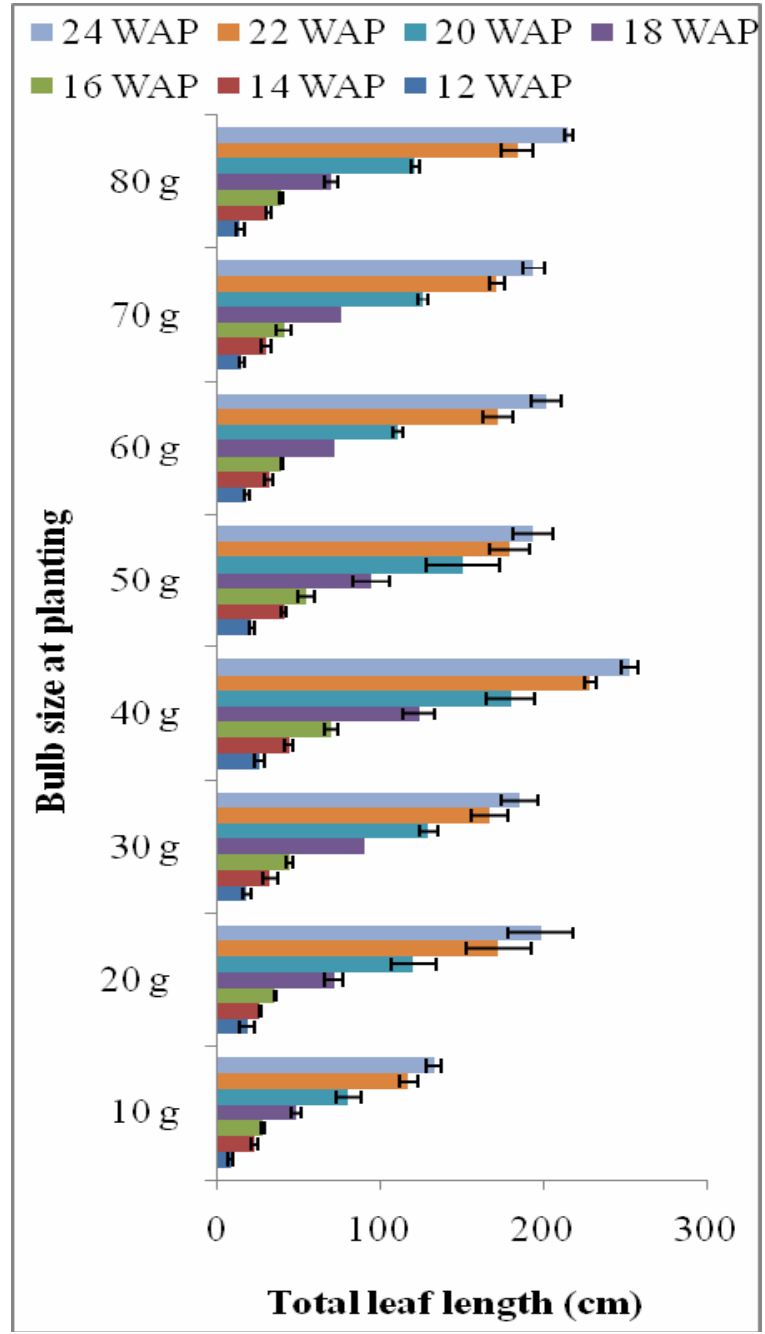

Fig 2: Effects of bulb size at planting on leaf growth of hyacinth: (a) total leaf width, and (b) total leaf length. Measurements were made on five plants per treatment (bulb size at planting) and the results shown are mean values \pm standard errors. 
(a)

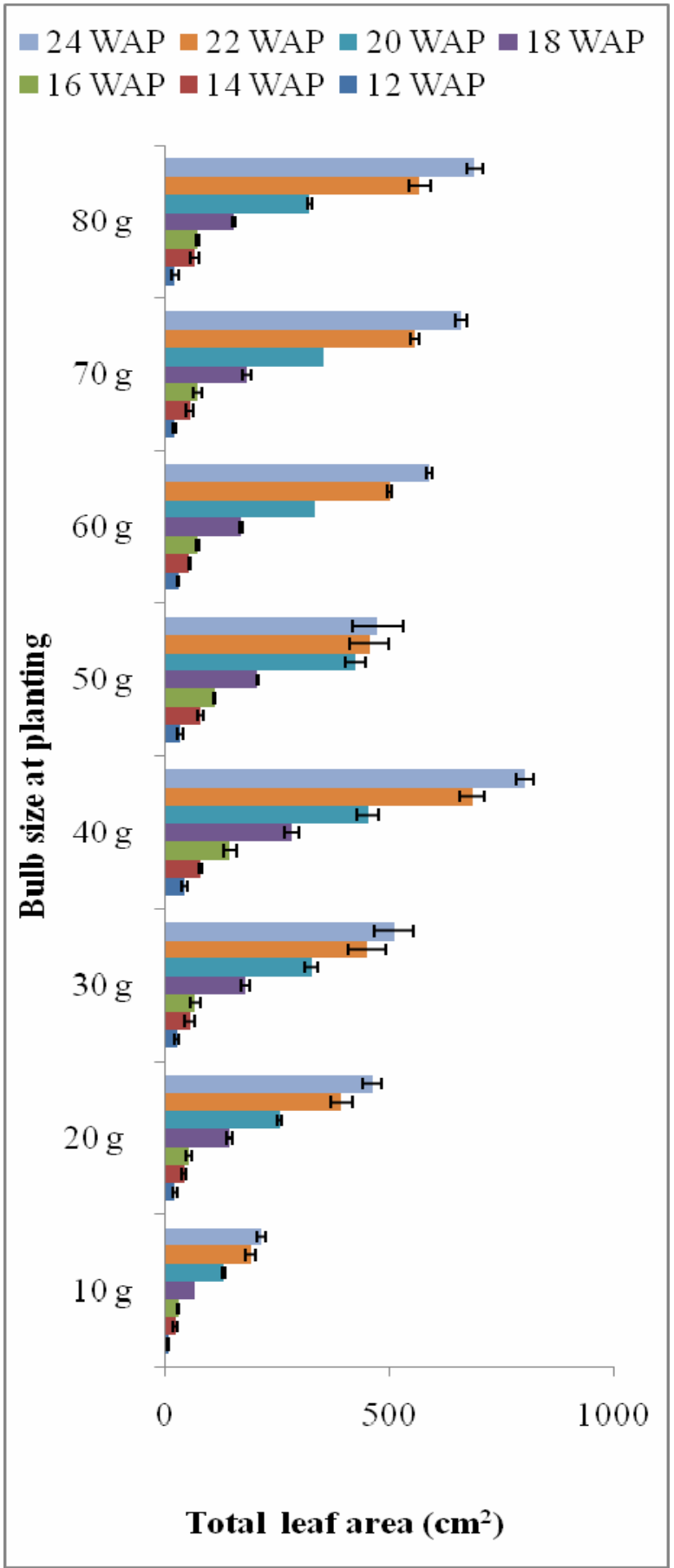

(b)

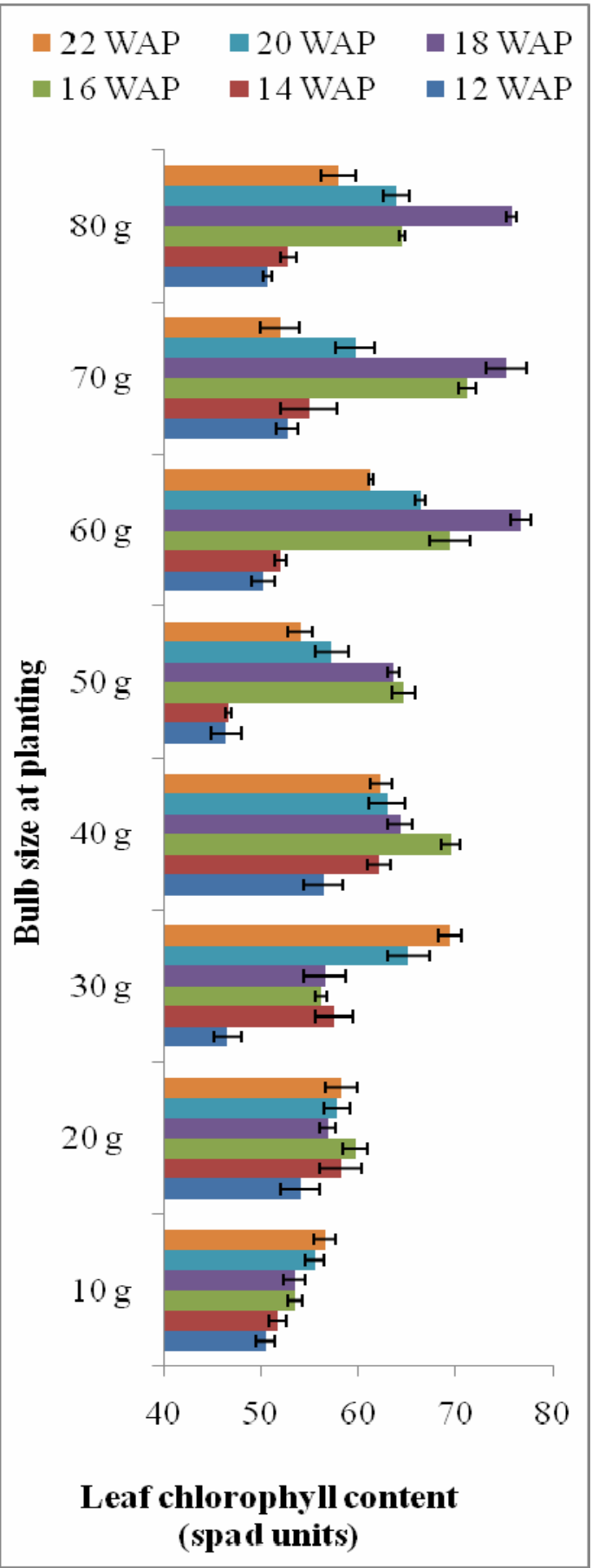

Fig 3: Influence of bulb size at planting on: (a) total leaf area and (b) chlorophyll content of hyacinth. Results are mean values \pm standard errors of five plants per treatment. 
(a)

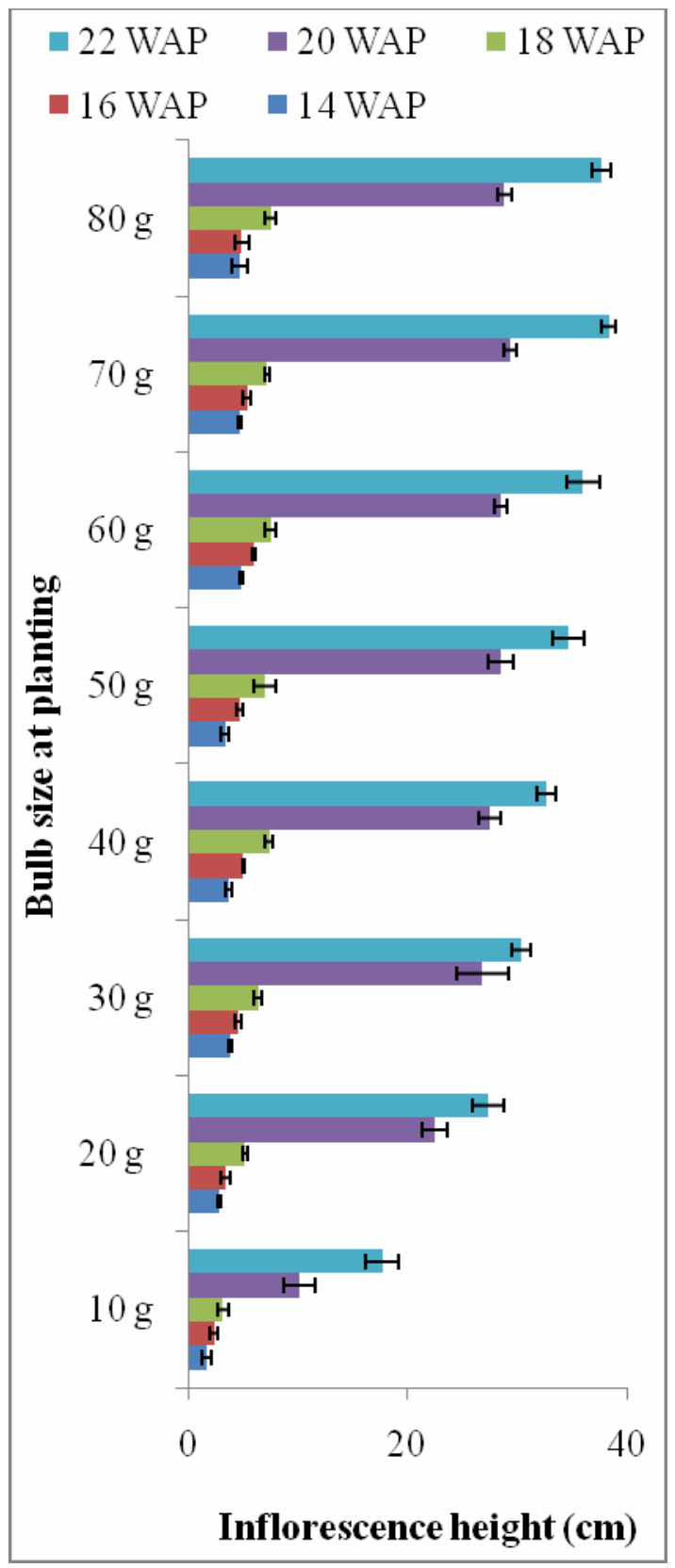

(b)

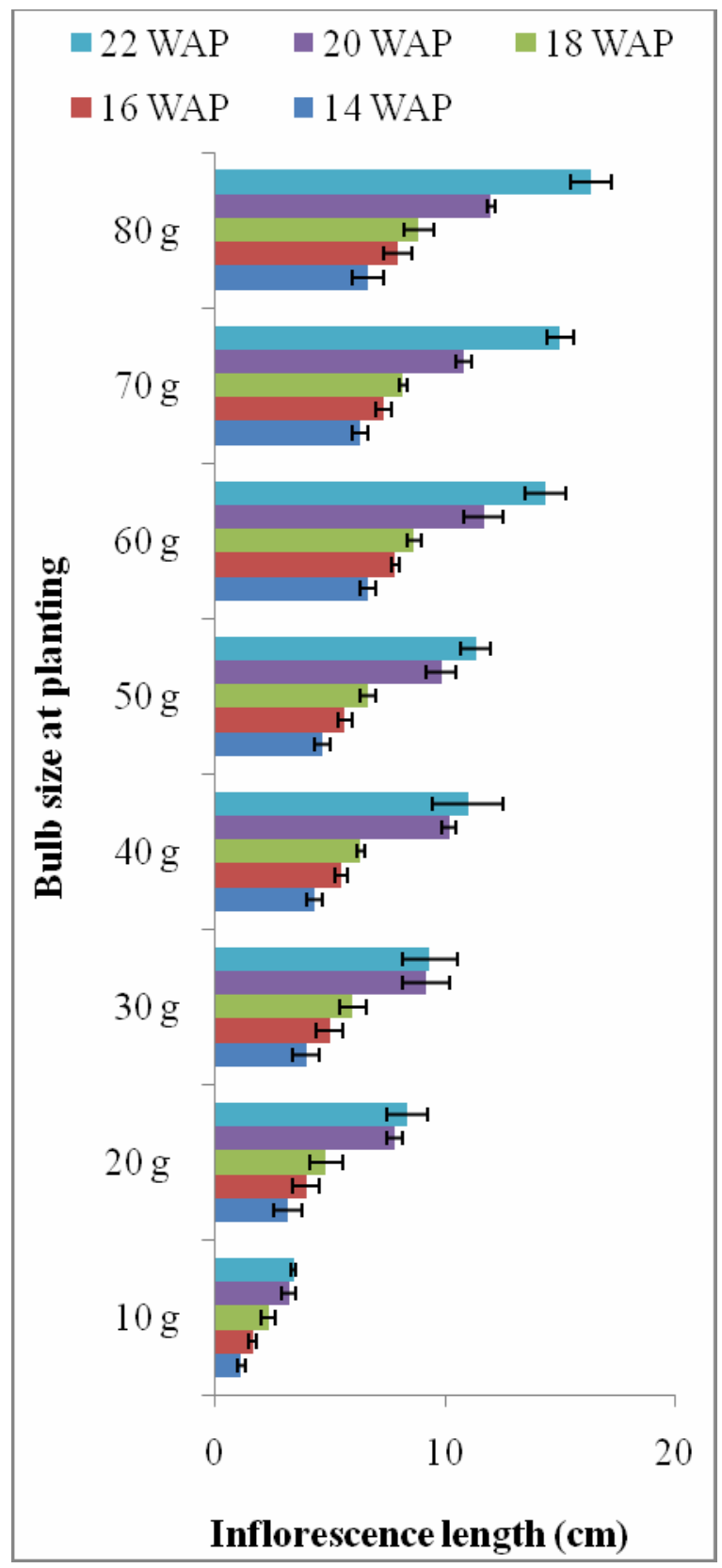

Fig 4: Influence of bulb size at planting on hyacinth inflorescence development: (a) inflorescence height and (b) inflorescence length. Values are means \pm standard errors of five plants per bulb size. 
(a)

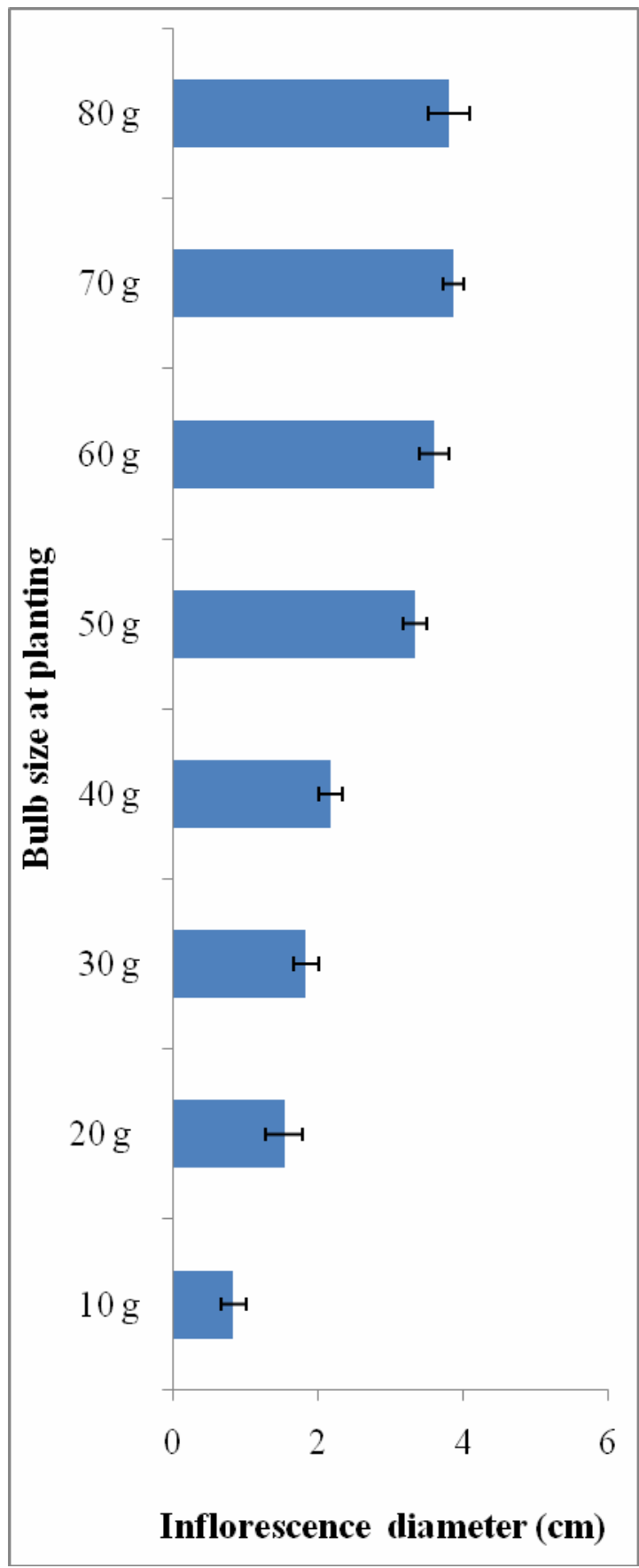

(b)

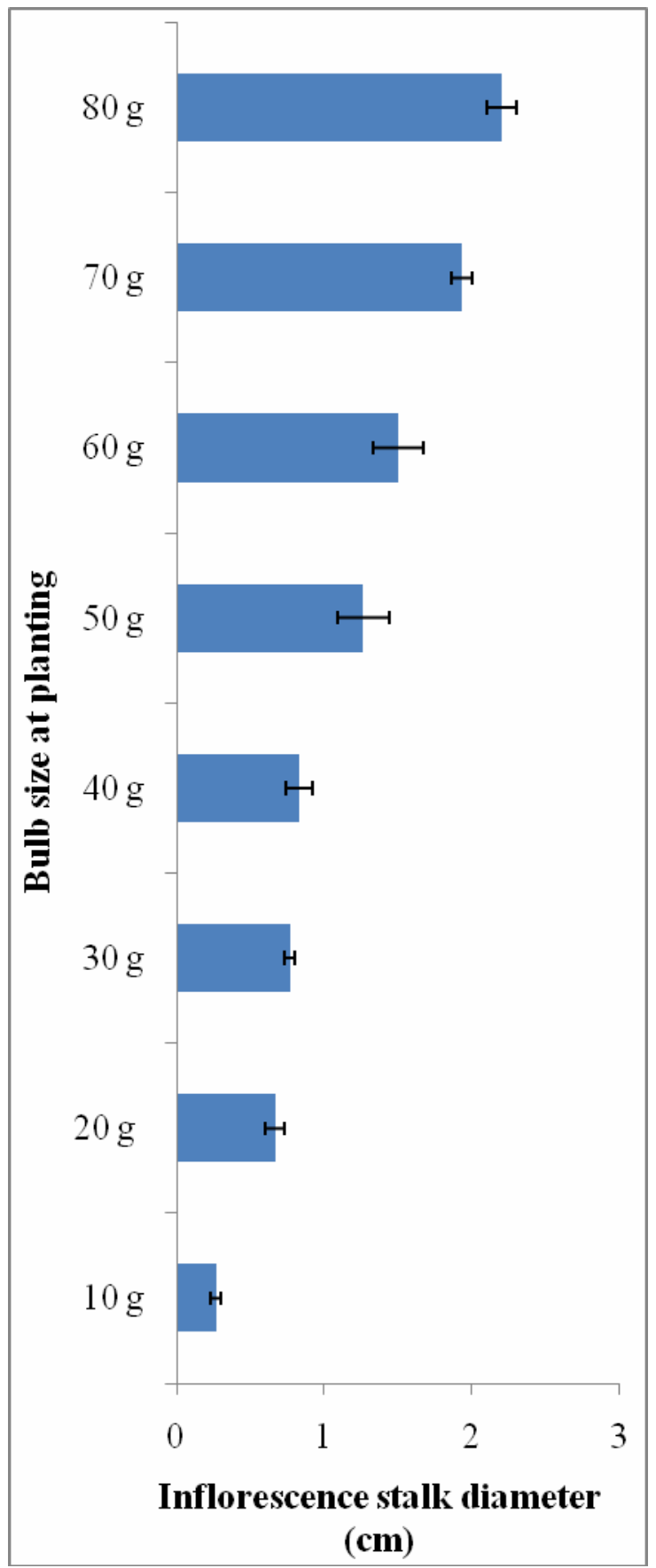

Fig 5: Bulb size at planting effects on: (a) inflorescence diameter and (b) inflorescence stalk diameter of hyacinth. Values shown are means \pm standard errors calculated from five plants per bulb size. 
(a)

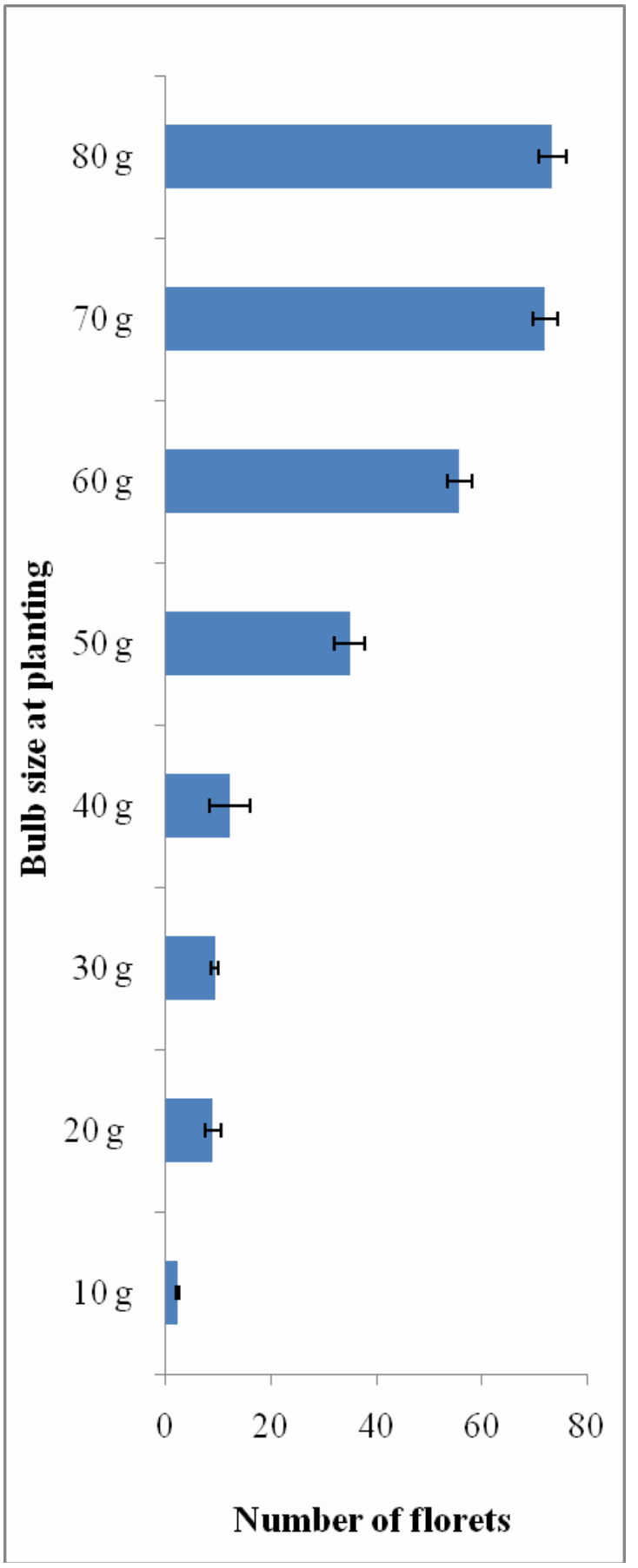

(b)

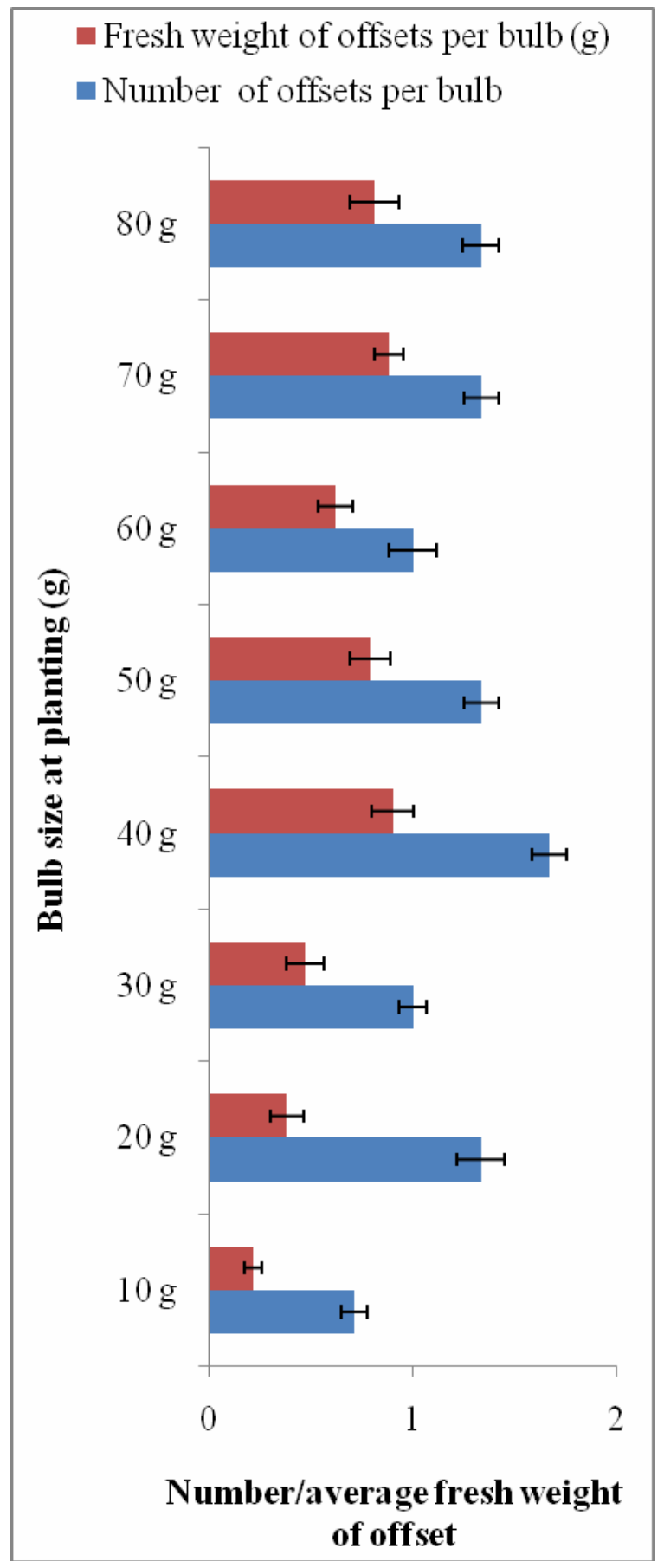

Fig 6: (a) Number of florets, and (b) bulblets formation in hyacinth as influenced by bulb size at planting. Results are means \pm standard errors of five replicates for each bulb size. 
(a)

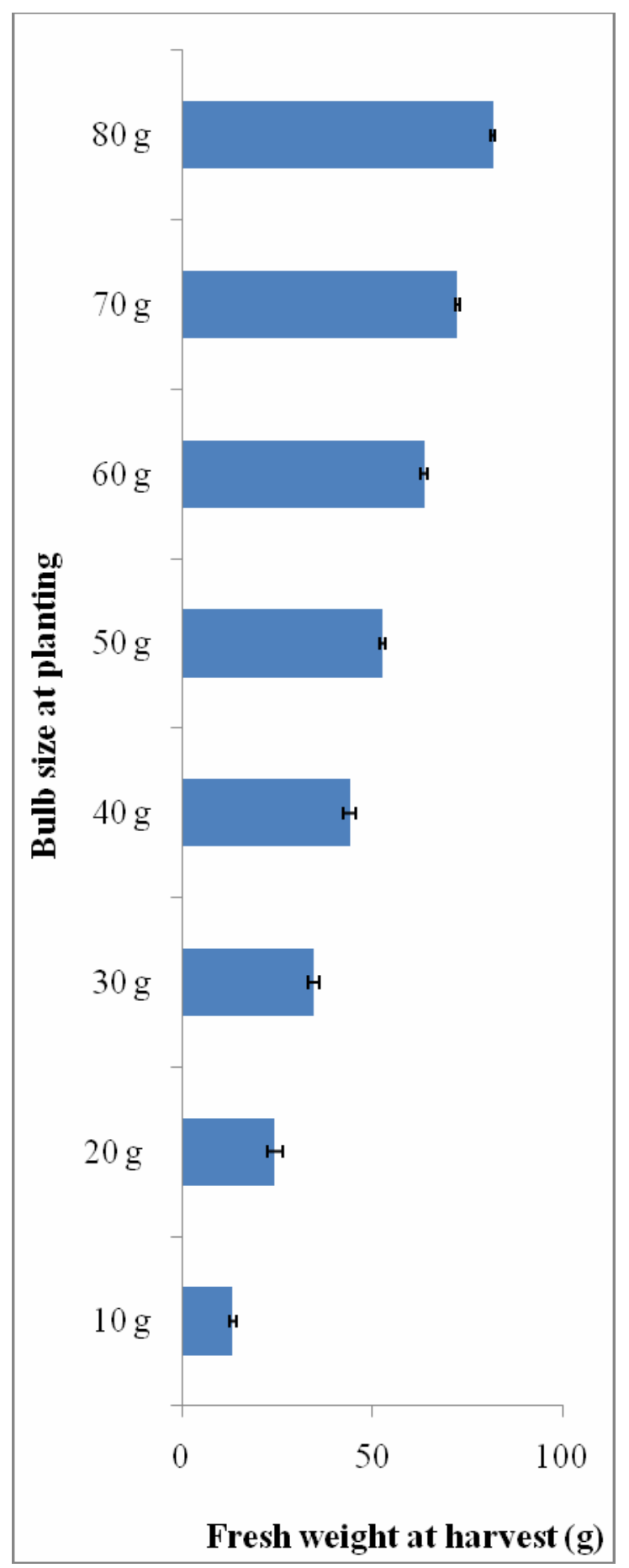

(b)

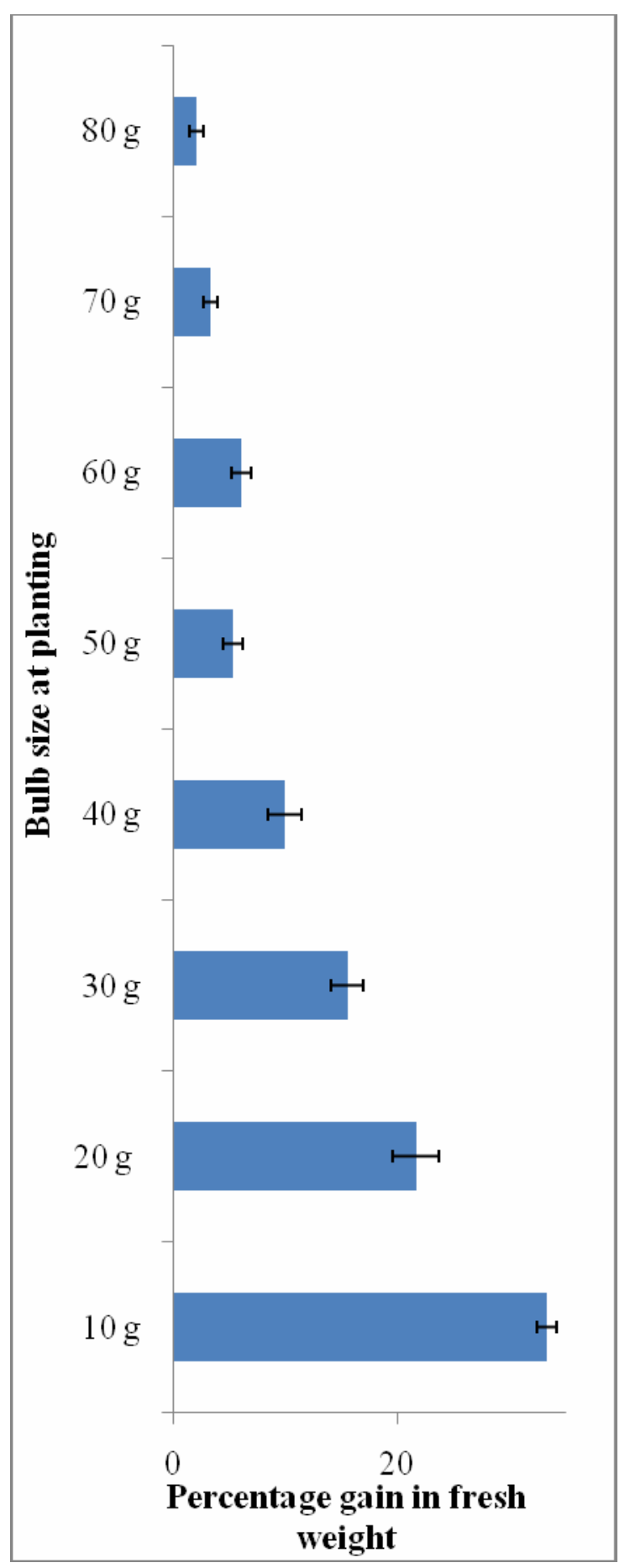

Fig 7: Influence of bulb size at planting of hyacinth on harvest yield, (a) Fresh weight of mother bulb at harvest and (b) percentage gain in fresh weight of the mother bulb at harvest. Bars represent mean values \pm standard error of five replicates. 
(a)

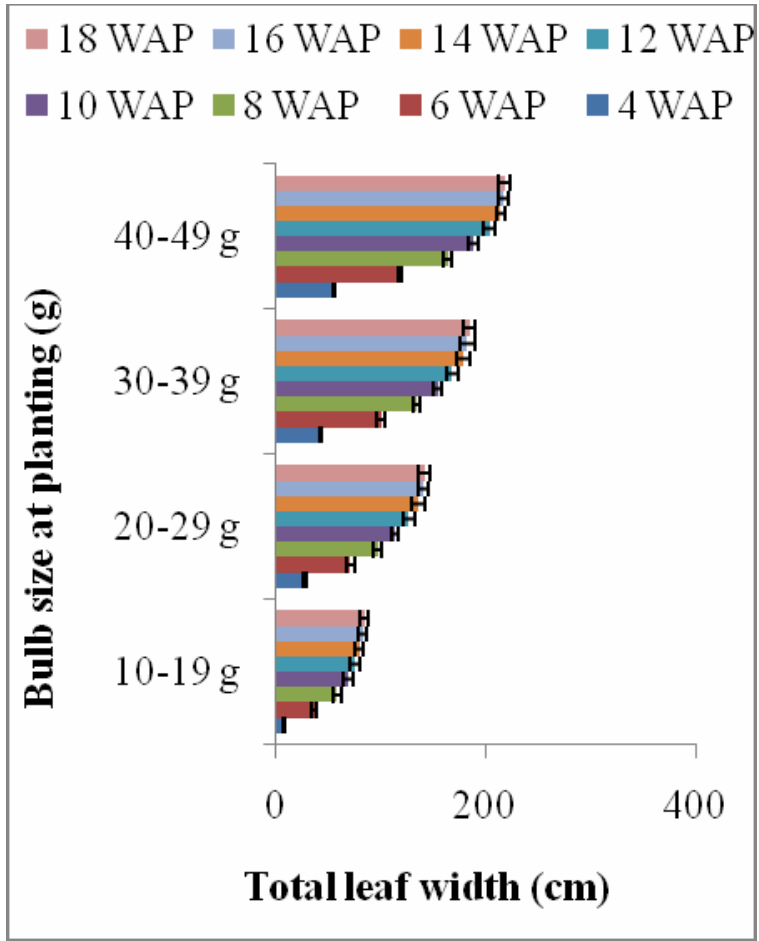

(c)

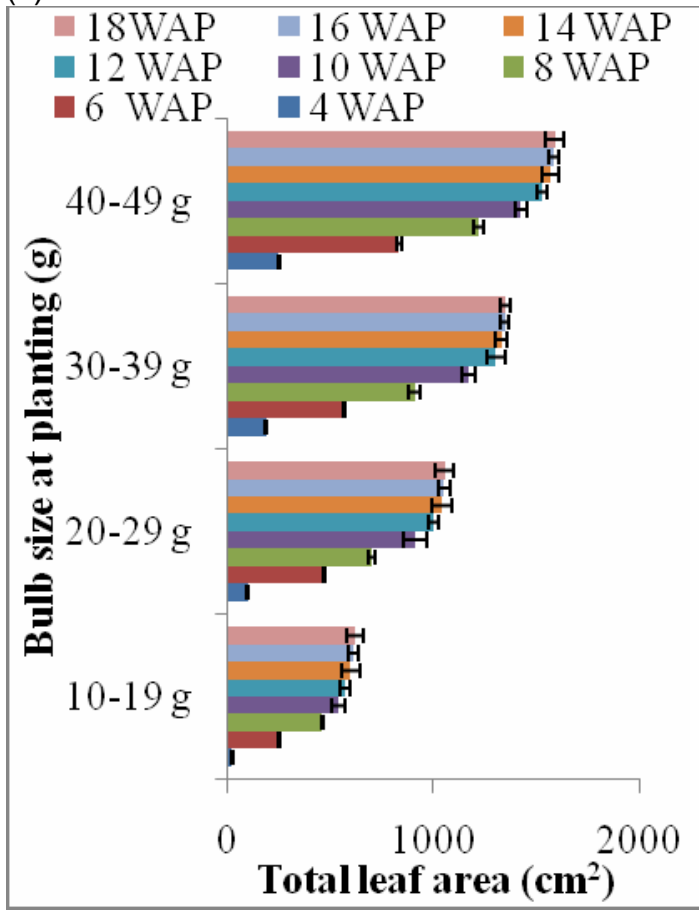

(b)

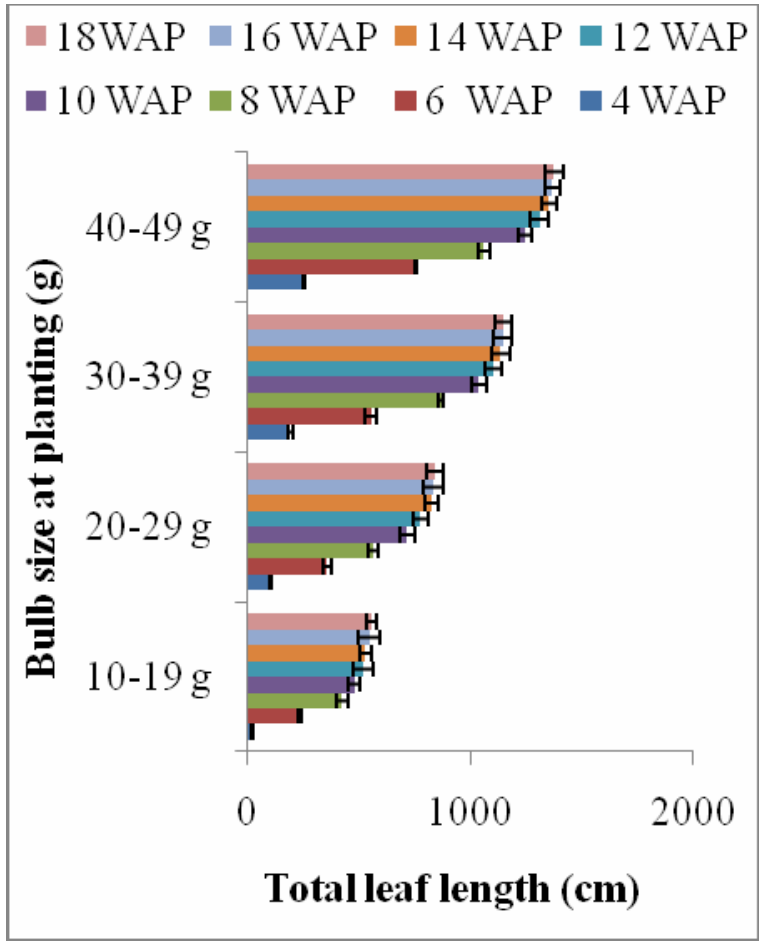

(d)

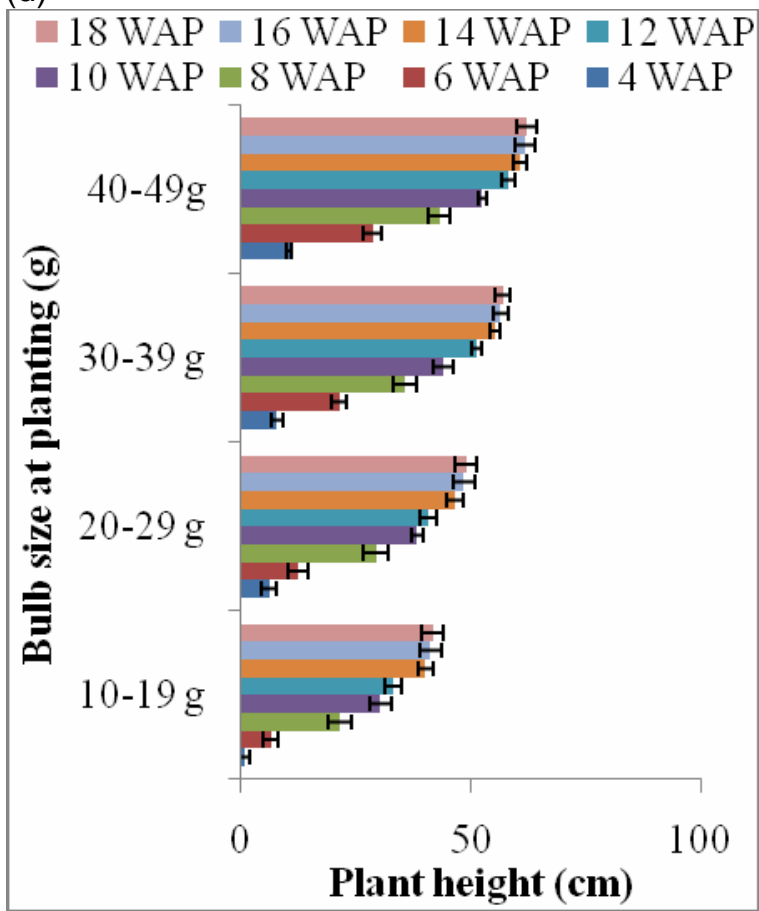

Fig 8: Impact of bulb size at planting on vegetative growth of the lily bulb, (a) total leaf width, (b) total leaf length, (c) total leaf area and (d) plant height. Values are means \pm standard error calculated from three plants. 
(a)

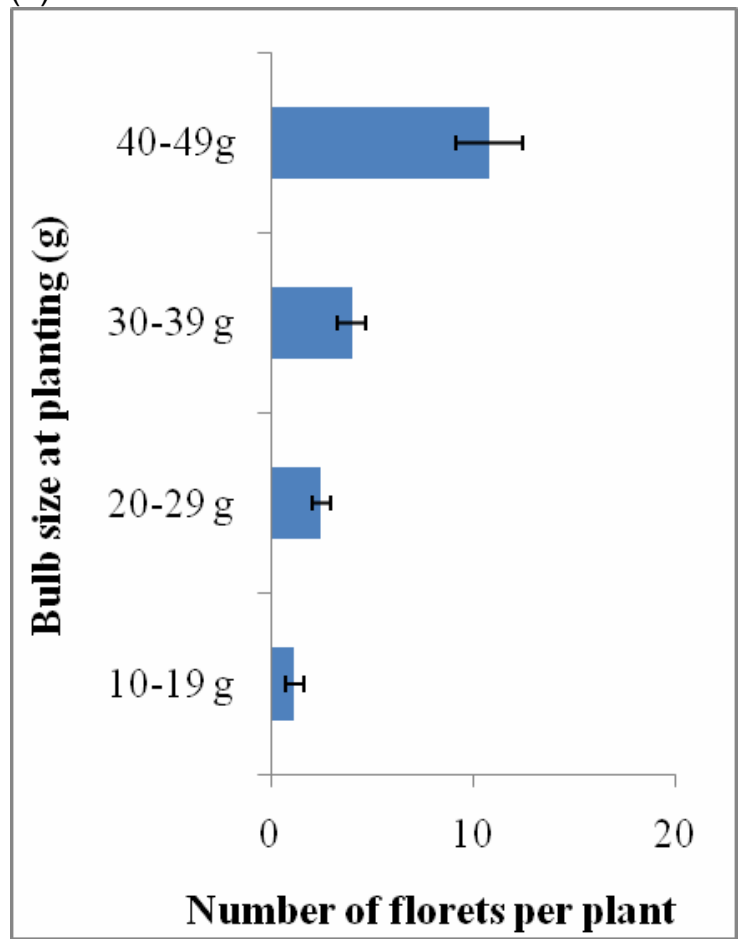

(c)

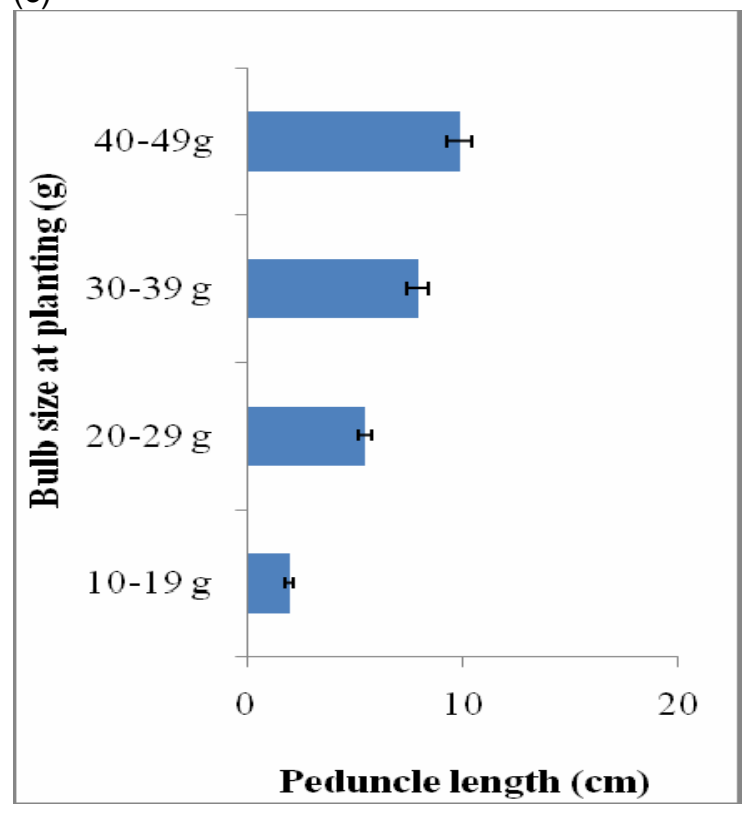

(b)

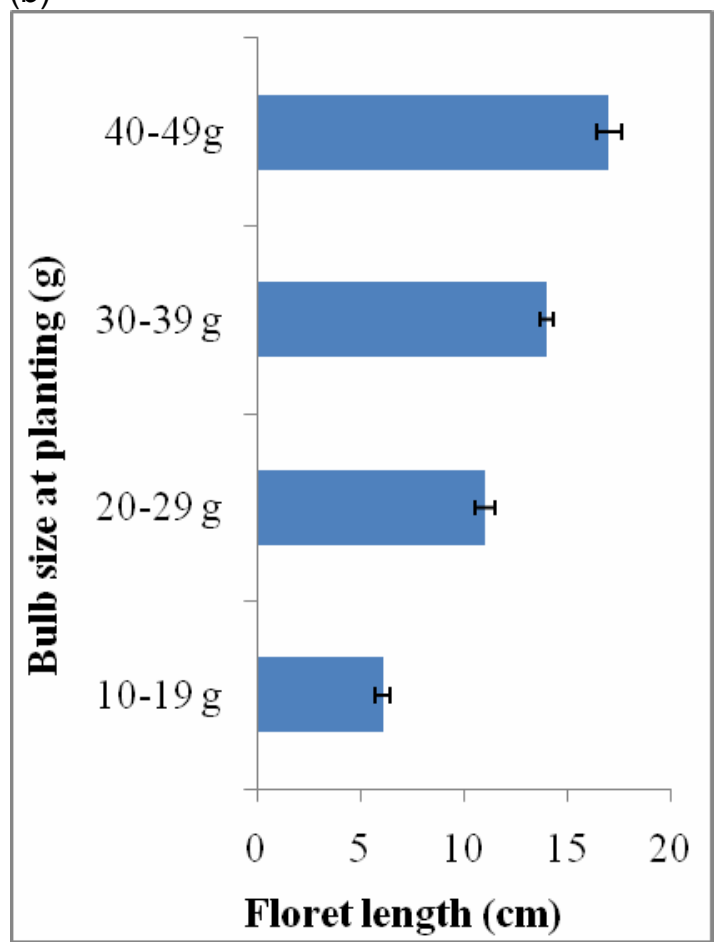

(d)

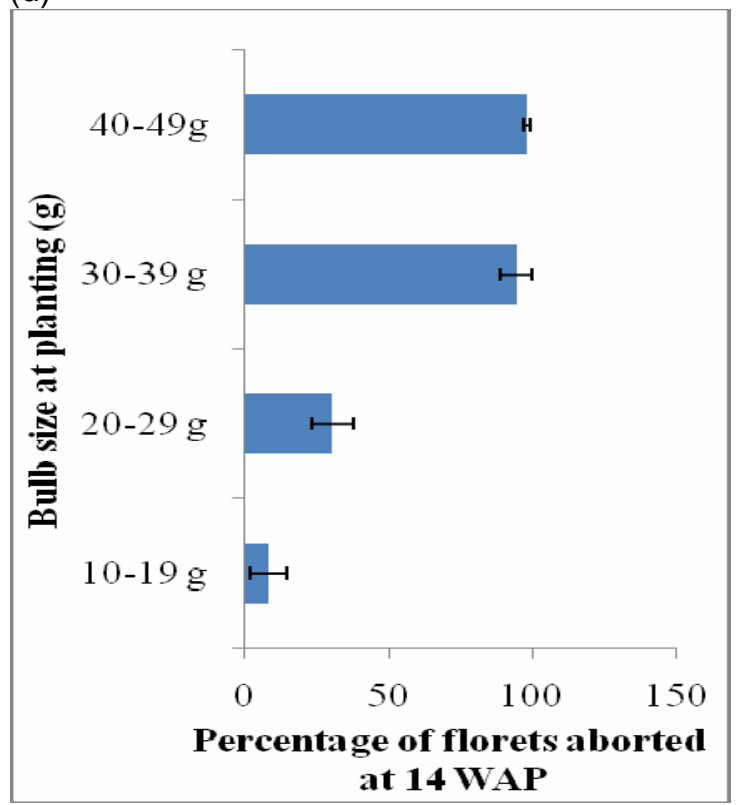

Fig 9: Effects of bulb size at planting on flower development of the lily bulb, (a) number of florets per plant, (b) floret length, (c) length of the peduncle, and (d) percentage abortion of florets. Results are mean values \pm standard errors of three plants. 
(a)

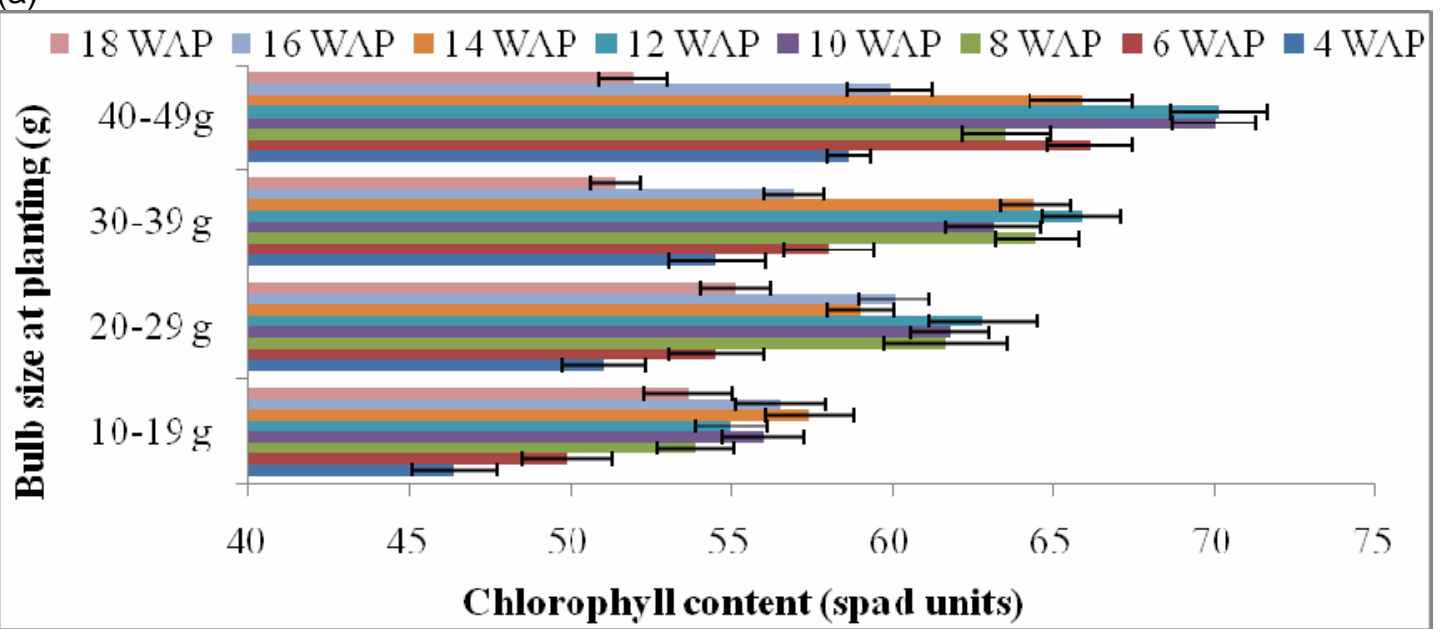

(b)

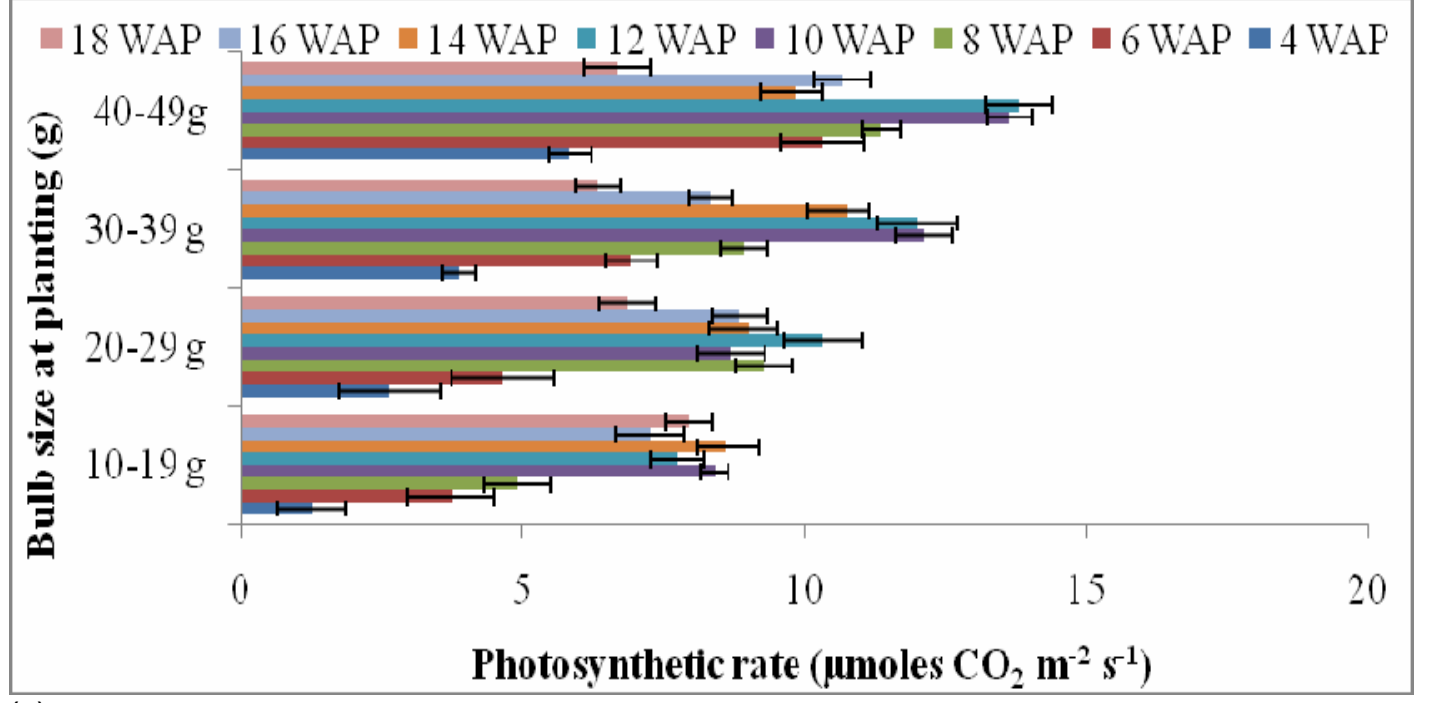

(c)

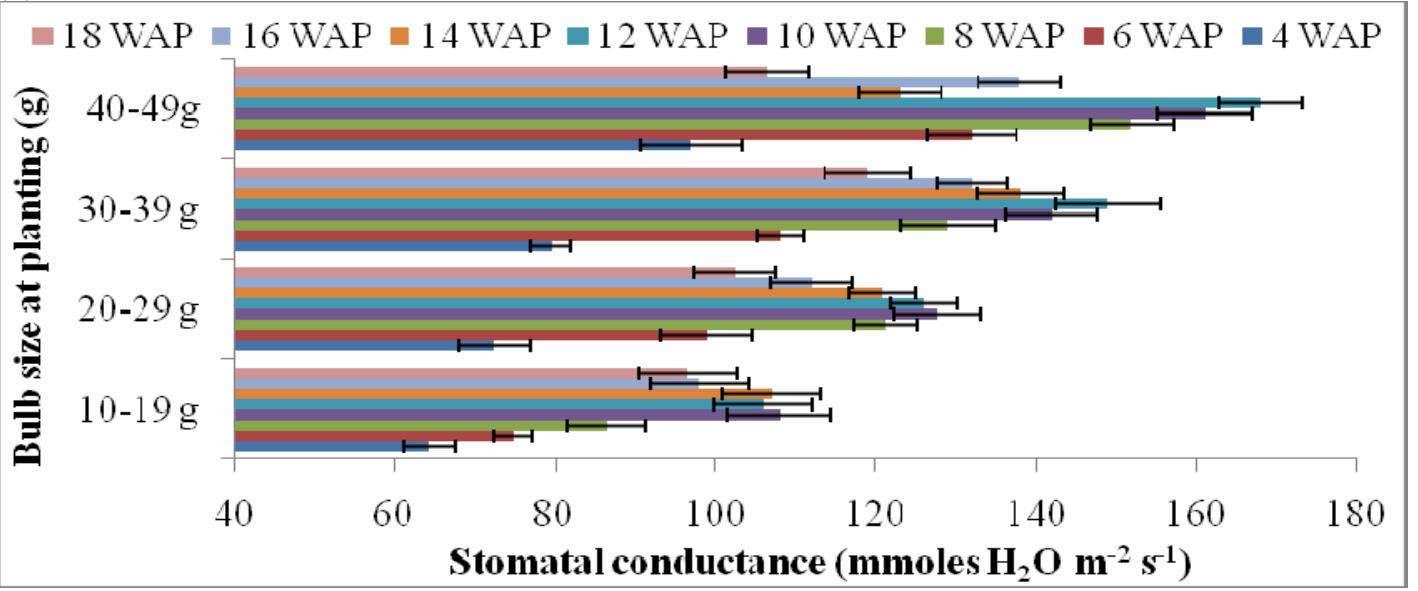

Fig 10: Bulb size at planting effects on (a) chlorophyll content, (b) photosynthetic rate and (c) stomatal conductance of the lily bulb. Values are means \pm standard error of three plants. 
Agric. Biol. J. N. Am., 2011, 2(2): 298-314

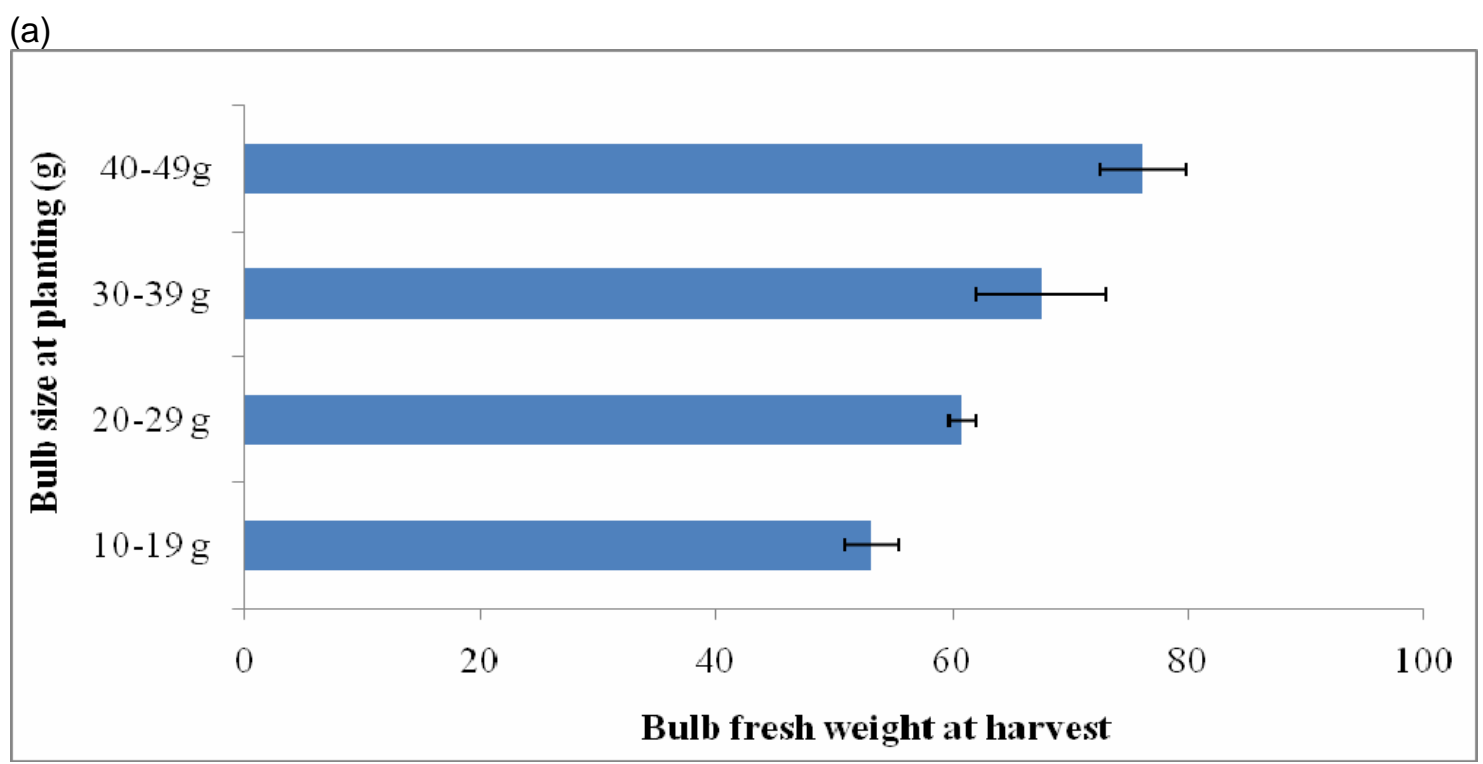

(b)

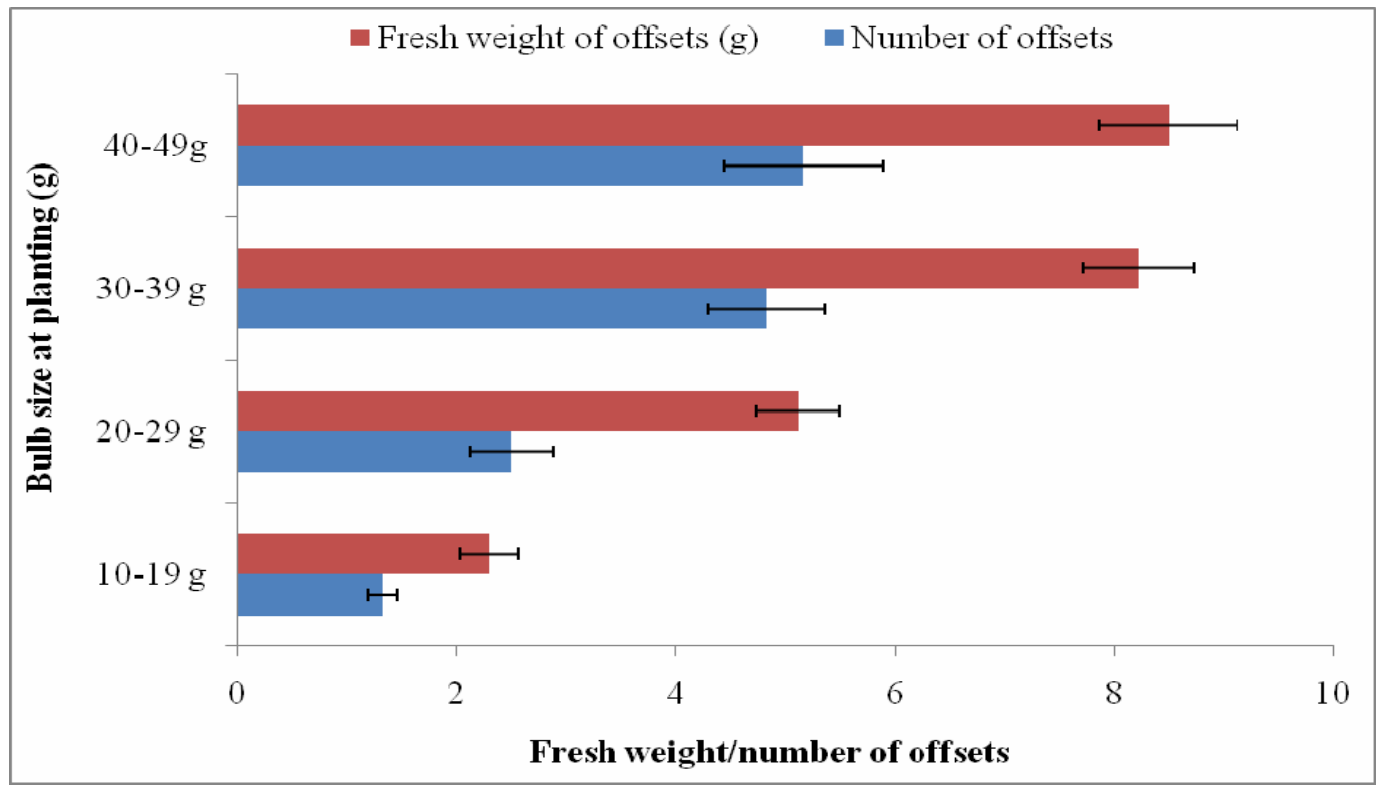

Fig 11: Influence of bulb size at planting on (a) fresh weight of the mother bulb and (b) offsets formation in the lily bulb. Results are the mean values \pm standard errors calculated from three replicates.

The lily: Results from the lily experiment did not differ significantly from those of the hyacinth experiment because the vegetative growth as well as all other parameters measured in the case of the lily increased with increasing bulb size at planting. Rate of leaf growth and plant height were high from 4 to 6 weeks after planting but between 6 and 12 weeks after planting growth rate was low. From 12 to 18 weeks after planting, growth had almost stopped as the increase in growth was not significant (Fig 8a, b, $c$ and d). For plants grown from the small bulbs (10$19 \mathrm{~g}$ bulb sizes), values of leaf width, leaf length, leaf area and plant height, at 4 weeks after planting were $7.56, \mathrm{~cm}, 22.89 \mathrm{~cm}, 20.56 \mathrm{~cm}^{2}$ and $0.83 \mathrm{~cm}$, respectively, whilst at 18 weeks after planting, values of these parameters were $83.93 \mathrm{~cm}, 556.80 \mathrm{~cm}$, 
$617.81 \mathrm{~cm}^{2}$ and $41.69 \mathrm{~cm}$. These represent relative growth rates of $0.779 \mathrm{~cm}^{-1 a y^{-1}}, 5.448 \mathrm{~cm}^{-1} \mathrm{yy}^{-1}, 6.094$ $\mathrm{cm}^{2}$ day $^{-1}$ and $0.417 \mathrm{~cm}$ day $^{-1}$, respectively. For plants obtained from the large bulbs (40-49 $\mathrm{g}$ bulb sizes), total leaf width, length, area and plant height were $55.32 \mathrm{~cm}, 250.7 \mathrm{~cm}, 248.02 \mathrm{~cm}^{2}$ and $10.50 \mathrm{~cm}$ at 4 weeks after planting, whilst at 18 weeks after planting, the values were $218.15 \mathrm{~cm}, 1374.5 \mathrm{~cm}$, $1589.50 \mathrm{~cm}^{2}$ and $62.20 \mathrm{~cm}$, respectively, giving relative growth rates of $1.662 \mathrm{~cm}^{-1 a y}{ }^{-1}, 11.467 \mathrm{~cm}$ day $^{-1}, 13.689 \mathrm{~cm}^{2}$ day ${ }^{-1}$ and $0.528 \mathrm{~cm}$ day $^{-1}$, respectively. Flower quality (number of florets, florets length and peduncle length) also increased with increasing bulb size at planting and in fact, the difference between values of plants produced from the small and large bulbs of these parameters was vast. For instance, plants grown from the small bulbs $(10-19 \mathrm{~g})$ recorded average values of $1.17 \mathrm{~cm}, 6.08$ $\mathrm{cm}$ and $1.95 \mathrm{~cm}$ for number of florets, floret length and peduncle lengths respectively, while those from the large bulbs (40-49 g) had 10.83, $17 \mathrm{~cm}$ and 9.83 $\mathrm{cm}$ of these parameters, respectively, (Fig $9 \mathrm{a}, \mathrm{b}$ and c). However, at 14 weeks after planting, the plants from the large bulbs aborted a higher percentage of florets than did those from the small bulbs (Fig 9d). As was observed in the case of hyacinth, chlorophyll concentration of plants from the large bulbs of the lily was higher than those of the small bulb, but in general, the reduction in chlorophyll content from 12 to 18 weeks after planting was higher for plants obtained from large bulbs than those from the small bulbs. A similar observation was made with the rate of photosynthesis and stomatal content (Fig 10a, b and c). Fresh weight of the mother bulb at harvest as well as the percentage gain in weight followed the same distribution pattern as that of hyacinth (Fig 11a). The fresh weight and number of daughter bulbs (bulblets) observed at harvest increased with increasing bulb size at planting (Fig 11b).

\section{DISCUSSION}

The study revealed that the size of the bulb used for planting is very important in the selection of planting materials for bulb production. This is because bulb size influenced vegetative growth both in the hyacinth and the lily. In general, as the size of the planted bulb increased, vegetative growth (leaf length, width, area and plant height) also increased. A similar observation was made by Rees (1969) in tulips and Burton (1966) in potatoes. The increase in magnitude of these parameters in the present study in proportion to the bulb size used for planting may be attributed to the amount of reserves stored in the bulb prior to planting. Our earlier work on these flower bulbs on carbohydrate regulation and partitioning (Manuscript under review) highlighted the role of reserve carbohydrates of bulbous plants in their growth and development. That is, the initial growth of these geophytes mainly depends on reserve materials stored in the bulb scales because these plants store and remobilise the reserved metabolites, mainly carbohydrates for their own growth and development. In general, since large bulbs are expected to have larger amounts of carbohydrates and other reserves than smaller bulbs, the former should therefore have better growth and development than the latter. Of course, the smallest bulb sizes behaved just like the peeled bulbs whose bulb reserves are reduced as a result of scale removal with the result that their growth and development is adversely affected (See paper on influence of scale removal on growth and development of these flower bulbs by the same authors, manuscript under review). Our results presented here have emphasized that reserve carbohydrates play a crucial role in the growth and development of bulbs. It also means that the performance of geophytes and other plants propagated by vegetative reproduction are influenced by the amount of stored reserves present at the time of planting (Bremner and El Saeed, 1963; Burton, 1966). However, contrary to Rees (1969) observation that small bulbs grew faster than big bulbs in tulips, results from the present study revealed that in either hyacinth or lilies, large bulbs recorded higher relative growth rate than small bulbs. Hidekazu et al. (1998) also observed that when larger tuber fragment is used as seed yam for propagation, the initial growth rate was higher than when small tuber fragment was used for yam production. The observed increase in relative growth rate of the large bulbs as compared with the small ones, according to Hidekazu et al. (1998), is because, in vegetatively propagated plants, large planting materials are characterised by higher and more conspicuous reduction in weight during sprouting than small bulbs and the higher the reduction in weight during sprouting, the higher the growth rate and this is what was also observed in this study.

In both hyacinth and the lily, chlorophyll content of plants produced from the large bulbs was generally higher than that obtained from the small bulbs. In hyacinth, plants from the small bulbs $(10-30 \mathrm{~g})$, maintained values of chlorophyll content from 12 to 22 weeks after planting whilst plants from the medium bulb (40-50 g) and the large bulbs (60-80 g) recorded a drop in this parameter at 18 weeks after 
planting. Thus in both hyacinth and the lily, the drop in chlorophyll concentration of plants produced from the large bulbs was higher than the medium bulbs; whilst the small bulbs maintained their chlorophyll concentration during the time that this parameter was measured. The reduction in chlorophyll content of plants from the large and medium bulbs after 18 weeks of growth as compared to those of the small ones, among other factors would be attributed to the natural process of ageing (Volaire, 2002) that occurred earlier in the case of plants produced from the large and medium bulbs, than plants obtained from the small bulbs. In general, the importance of chlorophyll in plants is mainly to absorb the light energy without which photosynthesis cannot proceed. It means that the amount of this pigment found in the plant is a measure of rate of photosynthesis and a deficiency or a drop in chlorophyll content of plants may adversely affect the rate of photosynthesis. It implies that plants from small bulbs would have the ability to maintain their photosynthetic rate for a longer time than plants produced from large bulbs. Apart from influencing the rate of photosynthesis, chlorophyll content of plants is an indicator of plant vigour. Therefore, a low chlorophyll content of plants may be as a result of a stress resulting from drought, disease infestation or mineral deficiency. In addition, a low chlorophyll concentration of plant may imply that the natural process of ageing (i.e. senescence) in plants is occurring. Since bulbs of different sizes were planted on the same type of soil, and no diseases were detected during the growth of the bulbs, and also no stresses were imposed on the plants, a reduction in chlorophyll of any of the treatments (plants produced from the different bulb sizes at planting) was assumed to be the result of senescence. During senescence, the rate of photosynthesis reduces and this was the behaviour of plants produced from large and medium bulbs. Hyacinth is a spring flowering bulb whilst lily is a summer bulb, and in this study, these two groups of bulbs shared similar characteristics in terms of vegetative growth and chlorophyll development, in relationship with the size of the planted bulbs. The above observations imply that a plant produced from large bulb grows faster to complete their life cycle but ages more quickly than that from small bulbs. Also during senescence, not only does chlorophyll concentration decrease but in general, photosynthetic rate as well as stomatal conductance of the plant also reduces. In hyacinth, photosynthetic rate and stomatal conductance of plants produced from large and small bulbs were not measured due to equipment breakdown and failure at the time of data collection. But in the lily, measurements of chlorophyll, photosynthetic rate and stomatal conductance showed that reduction in these parameters especially towards the end of the season was higher in plants from the large bulbs than those from the small bulbs. The higher percentage of florets aborted in the case of plants produced from the large bulbs compared with the proportion of florets aborted from plants produced from small bulbs in the lily is also an indication that plants produced from large bulbs mature quickly and reach senescence (Volaire, 2002) in flowering bulbs than those obtained from small bulbs.

The study also revealed that in both hyacinth and the lily, flower quality (inflorescence height, length, diameter, and number of florets, florets length and peduncle length) increased with increasing bulb size. This agrees with the observation made by De Munk and Schipper (1993) who also worked on Iris bulb and reported that weight was an important indicator for flowering in that species. Flowers, florets or inflorescence are important sink organs in flowering bulb that depend on the reserves stored in the bulb for their initial growth and development. Large bulbs have higher reserves than small bulbs and therefore plants produced from the former should have better flower quality than small bulbs. The same reason also explains why higher fresh weight of the mother bulb or bulblets was recorded of plants obtained from large bulbs as compared to those from the small sized bulbs. Results of the hyacinth experiment indicated that, the possession of a large bulb has little advantage, overall, to the plant in terms of vegetative growth and flower production. This is because after the $50 \mathrm{~g}$ bulb size, the results looked markedly similar. It means therefore that the extra carbohydrate reserves of plants produced from the large bulbs (> $50 \mathrm{~g}$ bulb sizes) were not used for vegetative growth. Maybe, this extra carbohydrate might be useful later during the life cycle of the bulb for survival during unfavourable environmental conditions. Working on Brodiaea, Han et al. (1991) observed that, growth and flower quality was independent of the mother corm size at the time of planting but it was the size of the apical meristem that determined the quality of the flowers produced. However, in the present study, growth and development depended on bulb size at planting, at least up to the $50 \mathrm{~g}$ bulb size. Maybe, the flower bulb uses the amount of reserves stored in the bulb prior to planting for its initial growth and development up to 
a certain threshold, or bulb size, in this case the $50 \mathrm{~g}$ bulb size. But above this size, growth and development may be determined by the size of the apical meristem. In this study, however, since measurements of the apical meristem was not made, the relationship between bulb size (carbohydrates reserves) and apical meristem in flower bulbs is not known, and this has not been documented in the literature.

\section{CONCLUSION}

It would be difficult to make a recommendation as to what bulb size is desirable during the planting of hyacinth or the lily unless the needs of the consumer or the aims of the grower are taken into consideration. This is because plants from large bulbs (50-80 $\mathrm{g}$ of hyacinth and $30-49 \mathrm{~g}$ in lily) generally grow well and produce better flower quality than those from small bulbs. Also, plants from large bulbs generally produce large bulbs at harvest and they also have better offsets formation as compared to small bulbs. However, any bulb size, particularly in the case of hyacinth, above $50 \mathrm{~g}$ will not give any results better than the $50 \mathrm{~g}$ bulb size in terms of vegetative growth and flower quality. Small bulbs retain chlorophyll and photosynthetic rate longer than large bulbs. Also, when weight gain is computed in terms of percentages, small bulbs produce higher weight gain at harvest than large ones. Thus for vegetative biomass and flower production or bulblets formation, growers should use bulbs that are not $>60$ $\mathrm{g}$ (hyacinth) and $>30 \mathrm{~g}$ (lily) as planting material. On the contrary, if the grower is interested in bulb yield (gain in fresh weight at harvest) and not vegetative biomass, flowers or bulblets, then any bulb size that is $<40 \mathrm{~g}$ (hyacinth) or $<30 \mathrm{~g}$ (lily) could be used for planting since these sizes will give high percentage gain in weight at harvest.

\section{REFERENCES}

Afonja, B. (1967). An analysis of an experiment with plots of different sizes. Trop. Agric., Trin. 44, 39-44.

Bremner, P. M. and Saeed, A. K. (1963). The significance of seed size and spacing. In The Growth of the Potato, eds. J. D. Ivins and F. L. Milthorpe, 367-80. Butterworths Sci. Publ., London.

Burton, W. G. (1966). The Potato. Veeman, Wageningen.

Chittendon, F. (1956). Comprehensive listing of species and how to grow them. Royal Horticultural Society Dictionary of Plants plus Supplement. Oxford University Press 1951.
Darkwa, A. A. (2008). Growth and biochemistry of European orchids. PhD Thesis, Biology, University of Sussex, UK.

De Munk, W.J. and Schipper, J. (1993). Iris-Bulbous and Rhizomatous. In: The Physiology of flower bulbs. De Hertogh, A. Le NARD, M. (Editors). Elsevier Scentia Publishers. Amsterdam: 349-379.

Enyi, B. A. C. (1967). Effect of spacing, sett size, ridging, and mulching on the development and yield of cocoyam (Xanthosoma tagittifolium Schott). Trop. Agric., Trin. 44, 53-60.

Fox. D. (1985). Growing Lilies. Croom Helm. The cultivation of the genus Lilium.

Gorer, R. (1970). The Development of Garden Flowers. London, Eyre and Spottiswode.

Grieve, (1984). A Modern Herbal. Penguin ISBN 0-14-046440-9.

Han S. Susan., Abraham H. Halevy, Roy M. Sachs, and Michael S. Reid. (1991). Flowering and Corm Yield of Brodiaea in Response to Temperature, Photoperiod, Corm Size, and Planting Depth. J. Amer. Soc. Hort. Sci. 116 (1):19-22. 1991.

Hedrick, U.P. (1972). Sturtevant's Edible Plants of the World. Dover Publications ISBN0-486-20459-6.

Hidekazu, T., Hironori T., Kenji, I., Masaki, T., Takao M. and Fumio, K. (1998). Effect of seed tuber size on the growth and development of Yam (Dioscorea alata L.). Jpn J Trop Agric. 42 (4), 282-287.

Huxley. A. (1992). The New RHS Dictionary of Gardening.. MacMillan Press 1992 ISBN0-333-47494-5.

Rees, A. R. (1969). Effect of Bulb Size on the Growth of Tulips. Ann. Bot. 33, 133-42, 1969.

Rees, A. R. (1972). The growth of Bulbs. Applied Aspects of the physiology of ornamental bulbous crop plants. Academic Press Inc. (London) Ltd.

Rees, A. R. (1985). Ornamental bulbous plants, p. 259307. In: A.H. Halevy (cd.). Handbook of flowering. vol. I. CRC Press, Boca Raton, Fla.

Usher. G. A. 1974. Medicinal uses of plants. Dictionary of Plants Used by Man. Constable ISBN 0094579202.

Volaire, F. (2002). Drought survival, summer dormancy and dehydrin accumulation in contrasting cultivars of Dactylis glomerata. Physiol Plantarum 116: 42-51.

Watad, A. A., Gidon Luria and Amihud Borochov. (1999). Aconitum: effects of environmental conditions and tuber size on growth, flowering and tuber production. Scientia Horticulturae 81 (1999) 135-147. 